\title{
Research Article \\ On the Global Asymptotic Stability of Switched Linear Time-Varying Systems with Constant Point Delays
}

\author{
M. de la Sen ${ }^{1}$ and A. Ibeas ${ }^{2}$ \\ ${ }^{1}$ Department of Electricity and Electronics, Faculty of Science and Technology, University of Basque, \\ Campus of Leioa (Bizkaia), Aptdo. 644, 48080 Bilbao, Spain \\ ${ }^{2}$ Department of Telecommunication and Systems Engineering, Engineering School, \\ Autonomous University of Barcelona, Cerdanyola del Vallés, 08193 Bellaterra, Barcelona, Spain
}

Correspondence should be addressed to M. de la Sen, manuel.delasen@ehu.es

Received 22 July 2008; Accepted 25 September 2008

Recommended by Antonia Vecchio

This paper investigates the asymptotic stability of switched linear time-varying systems with constant point delays under not very stringent conditions on the matrix functions of parameters. Such conditions are their boundedness, the existence of bounded time derivatives almost everywhere, and small amplitudes of the appearing Dirac impulses where such derivatives do not exist. It is also assumed that the system matrix for zero delay is stable with some prescribed stability abscissa for all time in order to obtain sufficiency-type conditions of asymptotic stability dependent on the delay sizes. Alternatively, it is assumed that the auxiliary system matrix defined for all the delayed system matrices being zero is stable with prescribed stability abscissa for all time to obtain results for global asymptotic stability independent of the delays. A particular subset of the switching instants is the so-called set of reset instants where switching leads to the parameterization to reset to a value within a prescribed set.

Copyright (C) 2008 M. de la Sen and A. Ibeas. This is an open access article distributed under the Creative Commons Attribution License, which permits unrestricted use, distribution, and reproduction in any medium, provided the original work is properly cited.

\section{Introduction}

Time-delay systems are receiving important attention in the last years. The reason is that they offer a very significant modeling tool for dynamic systems since a wide variety of physical systems possess delays either in the state (internal delays) or in the input or output (external delays). Examples of time-delay systems are war/peace models, biological systems, like, for instance, the sunflower equation, Minorsky's effect in tank ships, transmission systems, teleoperated systems, some kinds of neural networks, and so forth (see, e.g., [1-9]). Timedelay models are useful for modeling both linear systems (see, e.g., $[1-4,10]$ ) and certain nonlinear physical systems, (see, e.g., $[4,7-9,11]$ ). A subject of major interest in time-delay systems, as it is in other areas of control theory, is the investigation of the stability as well as 
the closed-loop stabilization of unstable systems, [2-4, 6-13] either with delay-free controllers or by using delayed controllers. Dynamic systems subject to internal delays are infinite dimensional by nature so that they have infinitely many characteristic zeros. Therefore, the differential equations describing their dynamics are functional rather than ordinary. Recent research on time delay systems is devoted to numerical stability tests, to stochastic timedelay systems, diffusive time-delayed systems, medical and biological applications [1417], and characterization of minimal state-space realizations [18]. Another research field of recent growing interest is the investigation in switched systems including their stability and stabilization properties. A general insight in this problem is given in [19-21]. Switched systems consist of a number of different parameterizations (or distinct active systems) subject to a certain switching rule which chooses one of them being active during a certain time. The problem is relevant in applications since the corresponding models are useful to describe changing operating points or to synthesize different controllers which can adjust to operate on a given plant according to situations of changing parameters, dynamics, and so forth. Specific problems related to switched systems are the following.

(a) The nominal order of the dynamics changes according to the frequency content of the control signal since fast modes are excited with fast input while they are not excited under slow controls. This can imply the need to use different controllers through time.

(b) The system parameters are changing so that the operation points change. Thus, a switched model which adjusts to several operation points may be useful [19-21].

(c) The adaptation transient has a bad performance due to a poor estimates initialization due to very imprecise knowledge of the true parameters. In this case, a multiparameterized adaptive controller, whose parameterization varies through time governed by a parallel multiestimation scheme, can improve the whole system performance. For this purpose, the parallel multiestimation scheme selects trough time, via a judicious supervision rule, the particular estimator associated with either the best identification objective, or the best tracking objective or the best mixed identification and tracking objectives. Such strategies can improve the switched system performance compared to the use of a single estimator/controller pair [5, 22].

This paper is devoted to the investigation of the global asymptotic stability properties of switched systems subject to internal constant point delays, while the matrices defining the delay-free and delayed dynamics are allowed to be time varying while fulfilling some standard additional regularity conditions like boundedness, eventual time differentiability, and being subject to sufficiently slow growing rates [23]. The various obtained asymptotic stability results are either dependent on or independent of the delay size and they are obtained by proving the existence of "ad-hoc" Krasovsky-Lyapunov functionals. It is assumed that either the current system matrix or that describing the system under null delay is stability matrices for results independent of and dependent on the delays sizes, respectively. This idea relies on the well-known fact that both of those matrices have to be stable for any linear time-invariant configuration in order that the corresponding time-delay system may be asymptotically stable, $[1,4,10]$, provided that a minimum residence time at each configuration is respected before the next switching to another configuration. The formalism is derived by assuming two classes of mutually excluding switching instants. The so-called reset-free switching instants are defined as those where some parametrical function is subject to a finite jump (equivalently, a Dirac impulse at its time derivative) which is not constrained to a finite set. The so-called reset switching instants are defined as those registering bounded jumps to values within some prescribed set of resetting parameterizations. The distinction between reset-free and reset switching instants 
is irrelevant for stability analysis since in both cases at least one parameter is subject to a bounded jump, or equivalently, to a Dirac impulse in its time derivative. Impulsive systems are of growing interest in a number of applications related, for instance, to very large forces applied during very small intervals of times, population dynamics, chemostat models, pest, and epidemic models, and so forth (see, e.g., [24-28] and references therein). However, it may be relevant in practical situations to distinguish a switch to prescribed time-invariant parameterizations (see the above situations (a)-(c)) from an undriven switching action. The paper is organized as follows. Section 2 is devoted to obtain asymptotic stability results dependent on the delay sizes. Section 3 gives some extension for global asymptotic stability independent of the delays. Numerical examples are presented in Section 4, where switching through time in between distinct parameterizations is discussed. Finally, conclusions end the paper. Some mathematical derivations concerned with the results of Sections 2 and 3 are derived in Appendix A.

\section{Asymptotic stability dependent on the delays}

Consider the $n$th order linear time-varying dynamic system with $q$ internal (in general, incommensurate) known point delays:

$$
\dot{x}(t)=\sum_{j=0}^{q} A_{j}(t) x\left(t-h_{j}\right)
$$

for any given bounded piecewise absolutely continuous function $\varphi:[-h, 0] \rightarrow \mathbf{R}^{n}$ of initial conditions, where $h:=\max _{1 \leq j \leq q}\left(h_{j}\right)$ with $h_{0}=0$, for some delays $h_{j} \in\left[0, \widehat{h}_{j}\right]$, of finite or infinite maximum allowable delays sizes $\widehat{h}_{j} \in \mathbf{R}_{0+}$, for all $j \in \bar{q}:=\{1,2, \ldots, q\}$, where $\mathbf{R}_{0+}$ is the nonnegative real axis $\mathbf{R}_{0+}:=\mathbf{R}_{+} \cup\{0\}=\{0 \leq z \in \mathbf{R}\}$; and $A_{j}: \mathbf{R}_{0+} \rightarrow \mathbf{R}^{n \times n}$, for all $j \in \bar{q} \cup\{0\}$. The following assumptions are made.

\subsection{Assumptions on the time-delay dynamic system (2.1)}

One or more of the following assumptions are used to derive the various stability results obtained in this paper.

Assumption 2.1. All the entries of the matrix functions $A_{j}: \mathbf{R}_{0_{+}} \rightarrow \mathbf{R}^{n \times n}$ are piecewise continuous and uniformly bounded for all $j \in \bar{q} \cup\{0\}$.

Assumption 2.2. All the eigenvalues $\lambda_{i}\left(\sum_{j=0}^{q} A_{j}(t)\right)$ of the matrix function $\sum_{j=0}^{q} A_{j}(t)$ satisfy $\operatorname{Re} \lambda_{i}\left(\sum_{j=0}^{q} A_{j}(t)\right) \leq-\rho_{0}<0$ for all $t \in \mathbf{R}_{0+}$, for all $i \in \bar{\sigma}(\sigma \leq n)$ for some $\rho_{0} \in \mathbf{R}_{+}:=\{0<z \in \mathbf{R}\}$, that is, $\sum_{j=0}^{q} A_{j}(t)$ is a stability matrix for all $t \in \mathbf{R}_{0+}$.

Assumptions 2.3. The matrix functions $A_{j}: \mathbf{R}_{0+} \rightarrow \mathbf{R}^{n \times n}$ are almost everywhere time differentiable with essentially bounded time derivative for all $j \in \bar{q} \cup\{0\}$ possessing eventual isolated bounded discontinuities, then $\operatorname{ess}^{\sup _{t \in \mathbf{R}_{0+}}}\left(\left\|\sum_{j=0}^{q} \dot{A}_{j}(t)\right\|\right) \leq \gamma_{\ell}<\infty$ with $\gamma_{\ell}$ being a $\ell$-norm dependent nonnegative real constant and, furthermore,

$$
\int_{t}^{t+T}\left\|\dot{A}_{j}(\tau)\right\| d \tau \leq \mu_{j} T+\alpha_{j} \leq \mu T+\alpha \quad \forall j \in \bar{q} \cup\{0\}
$$

for some $\alpha_{j}, \mu_{j}, \alpha, \mu \in \mathbf{R}_{+}$for all $t \in \mathbf{R}_{0+}$, and some fixed $T \in \mathbf{R}_{0+}$ independent of $t$. 
At time instants $t$, where the time-derivative of some entry of $A_{j}(t)$ does not exist for any $j \in \bar{q} \cup\{0\}$, the time derivative is defined in a distributional Dirac sense as $\dot{A}_{j}(t)=\Gamma(t) \delta(0)$ what equivalently means the presence of a discontinuity at $t$ in $A_{j}(t)$ defined as

$$
A_{j}\left(t^{+}\right)=A_{j}\left(t^{-}\right)+\lim _{\varepsilon \rightarrow 0^{+}} \int_{-\varepsilon}^{\varepsilon} \Gamma_{j}(\tau) \delta(t-\tau) d \tau=A_{j}(t)+\Gamma_{j}(t)
$$

Assumption 2.1 is relevant for existence and uniqueness of the solution of (2.1). The differential system (2.1) has a unique state-trajectory solution for $t \in \mathbf{R}_{+}$for any given piecewise absolutely continuous function $\varphi:[-h, 0] \rightarrow \mathbf{R}^{n}$ of initial conditions. This follows from Picard-Lindelöff existence and uniqueness theorem. Assumption 2.2 establishes that $\sum_{j=0}^{q} A_{j}(t)$ is a stability matrix for all time what is known to be a necessary condition for the global asymptotic stability of the system (2.1) for a set of prescribed maximum delays in the time-invariant case. It is well known that even if $A_{j} \equiv 0$ for all $j \in \bar{q}$, then the resulting linear time-varying delay-free system cannot be proved to be stable without some additional assumptions, like for instance, Assumptions 2.3. The latest assumption is related to the smallness of the time-derivative of the delay-free system matrix everywhere it exists or generating sufficiently small bounded discontinuities in $\sum_{j=0}^{q} A_{j}(t)$ at times, where it is impulsive. An alternative assumption to Assumptions 2.3 which avoids the assumption of almost everywhere existence of a bounded $\dot{A}_{j}(t)$, for all $j \in \bar{q} \cup\{0\}$ (see second part of Assumption 2.1) might be stated in terms of sufficiently smallness of $\left\|\Delta A_{j}(t)\right\|$ for $\Delta A_{j}(t):=$ $A_{j}(t)-A_{j}^{*}$ for all $t \in \mathbf{R}_{0+}$ for all $j \in \bar{q} \cup\{0\}$ for some constant stability matrix $\sum_{j=0}^{q} A_{j}^{*}$ whose eigenvalues satisfy $\operatorname{Re} \lambda_{i}\left(\sum_{j=0}^{q} A_{j}^{*}\right) \leq-\rho_{0}<0$. Such an alternative assumption guarantees also the global existence and uniqueness of the state-trajectory solution of (2.1) and it allows obtaining very close stability results to those being obtainable from the given assumptions. For global asymptotic stability dependent of the delay sizes on the first delay interval, the stability of the values taken by the matrix function $\left(\sum_{j=0}^{q} A_{j}(t)\right)$ is required within some real interval of infinite measure. Such an interval possesses a connected component being of infinite measure which is a necessary condition for global asymptotic stability for zero delays (see Theorem 2.12(i) ).

\subsection{Switching function, switching sequence, and basic assumptions on the switching matrix function}

Assumption 2.1 admits bounded discontinuities in the entries of $A_{j}(t)$ for $j \in \bar{q} \cup\{0\}$. At such times $A_{j}\left(t^{+}\right)$denote right values of the matrix function while $A_{j}\left(t^{-}\right)$is simply denoted by $A_{j}(t)$. A set of $p$ resetting systems of (2.1) is defined by the linear time-invariant systems:

$$
\dot{z}_{j}(t)=\left(\sum_{i=0}^{q} A_{i j}\right) z_{j}\left(t-h_{j}\right)
$$

for some given $A_{j i} \mathbf{R}^{n \times n}$ for all $j \in \bar{p}$ for all $i \in \bar{q} \cup\{0\}$ for some given $p \in \mathbf{N}$. Those parameterizations are used to reset the system (2.1) at certain reset instants defined later on. Assumption 2.2 extends in a natural fashion to include the resetting systems as follows. 
Assumption 2.4. All the eigenvalues $\lambda_{k}\left(\sum_{i=0}^{q} A_{i j}\right)$ satisfy $\operatorname{Re} \lambda_{k}\left(\sum_{i=0}^{q} A_{i j}\right) \leq-\rho_{0}<0$ for all $t \in \mathbf{R}_{0+}$ for all $k \in \bar{\sigma}_{j 0}\left(\sigma_{j 0} \leq n\right)$ for all $j \in \bar{p}$; that is, $\sum_{i=0}^{q} A_{i j}$ are constant stability matrices with prescribed stability abscissa $-\rho_{0}<0$ for all $j \in \bar{p}$.

The following definitions are then used.

Definition 2.5. The switching matrix function is a mapping $\sigma: \mathbf{R}_{0_{+}} \rightarrow\left\{\left(A_{j}\left(t^{+}\right)-A_{j}(t)\right), \forall j \in\right.$ $\bar{q} \cup\{0\}\} \subset \mathbf{R}^{n \times(q+1) n}$ from the nonnegative real axis to the set of real $n \times(q+1) n$ matrices.

The trivial switching matrix function is that being identically zero so that no switch occurs. If some switch occurs then the switching matrix function is nonzero. The switching matrix function is colloquially referred to in the following as the switching law.

Definition 2.6 (switching instant). $t \in \mathbf{R}_{0+}$ is a switching instant if $A_{j}\left(t^{+}\right) \neq A_{j}(t)$ for some $j \in \bar{q} \cup\{0\}$.

The set of switching instants generated by the switching law $\sigma$ is denoted by $\mathrm{ST}(\sigma)$. Two kinds of switching instants, respectively, reset instants and reset-free switching instants defined in Definitions 2.7 and 2.8 are considered.

Definition 2.7 (reset instant). $t \in \mathbf{R}_{0+}$ is a reset instant generated by the switching law $\sigma$ if $t \in \mathrm{ST}(\sigma)$ and $A_{i}\left(t^{+}\right)=A_{i j}$ for some $i \in \bar{q} \cup\{0\}$ and some $j \in \bar{p}$, provided that $A_{i}(t)=A_{i k} \neq A_{i j}$ for some $k(\neq j) \in \bar{p}$.

The set of reset instants generated by the switching law $\sigma$ is denoted by $\mathrm{ST}_{r}(\sigma)$. Note that $\mathrm{ST}_{r}(\sigma) \subset \mathrm{ST}(\sigma)$ from Definitions 2.6 and 2.7. Note also that the whole system parameterization is driven to some of the prefixed resetting systems (2.4) when a reset instant happens. Note that at reset instants, $\dot{A}_{i}(t)=\left(A_{i j}-A_{i}(t)\right) \delta(0)$ for some $i \in \bar{q} \cup\{0\}, j \in \bar{p}$.

Definition 2.8 (reset-free switching instant). $t \in \mathbf{R}_{0+}$ is a switching reset-free instant generated by the switching law $\sigma$ if $t \in \mathrm{ST}(\sigma)$ and $A_{i j} \neq A_{i}\left(t^{+}\right) \neq A_{i}(t)$ for some $i \in \bar{q} \cup\{0\}$, for all $j \in \bar{p}$.

The set of reset-free instants is denoted by $t \in \mathrm{ST}_{\mathrm{rf}}(\sigma)$. Note that at reset-free switching instants some of the switched system parameters suffer an undriven bounded discontinuity. If all the parameters jump to a parameterization (2.4) at the same time, then the corresponding instant is considered a reset time instant. Note that $\mathrm{ST}_{\mathrm{rf}}(\sigma) \subset \mathrm{ST}(\sigma)$, $\mathrm{ST}(\sigma)=\mathrm{ST}_{r}(\sigma) \cup \mathrm{ST}_{\mathrm{rf}}(\sigma)$, and $\mathrm{ST}_{r}(\sigma) \cap \mathrm{ST}_{\mathrm{rf}}(\sigma)=\varnothing$ from Definitions 2.6-2.8, that is, the whole set of switching instants is the disjoint union of the sets of reset and reset-free switching instants.

Definition 2.9. The partial switching sequence $\mathrm{ST}(\sigma, t)$, the partial switching sequence $\mathrm{ST}_{r}(\sigma, t)$, and the reset-free partial switching sequence $\mathrm{ST}_{\mathrm{rf}}(\sigma, t)$, generated by the switching law $\sigma: \mathbf{R}_{0+} \rightarrow\left\{\left(A_{j}(t)-A_{j}\left(t^{-}\right)\right), \forall j \in \bar{q} \cup\{0\}\right\} \subset \mathbf{R}^{n \times(q+1) n}$ up till any time $t \in \mathbf{R}_{0+}$, are defined, respectively, by ST $(\sigma, t):=\left\{t_{i} \in \mathrm{ST}(\sigma): t_{i}<t\right\}, \mathrm{ST}_{r}(\sigma, t):=\left\{t_{i} \in \mathrm{ST}_{r}(\sigma): t_{i}<t\right\}$, and $\mathrm{ST}_{\mathrm{rf}}(\sigma, t):=\left\{t_{i} \in \mathrm{ST}_{\mathrm{rf}}(\sigma): t_{i}<t\right\}$.

Remark 2.10. An interpretation of Assumptions 2.3 is that the following conditions hold for any given $\ell$-matrix norm for some nonnegative norm dependent real constants $\mu_{j}, \alpha_{j}, \mu$, and $\alpha$ for all $j \in \bar{q} \cup\{0\}:$ 


$$
\begin{aligned}
\operatorname{ess} \sup \left\|\dot{A}_{j}(t)\right\| & \leq \mu_{j} \leq \max \left(\underset{t \in \mathbf{R}_{0}^{+}}{\operatorname{ess} \sup }\left\|\dot{A}_{j}(t)\right\|: j \in \bar{q} \cup\{0\}\right) \leq \mu, \\
\left\|\sum_{\tau \in \mathrm{ST}(\sigma) \cap[t, t+T)} \dot{A}_{j}(\tau)\right\| & \leq \sum_{\tau \in \mathrm{ST}(\sigma) \cap[t, t+T)}\|X(\tau) \delta(0)\| \leq \alpha_{j} \\
& \leq \max \left(\left\|\sum_{\tau \in \mathrm{ST}(\sigma) \cap[t, t+T)} \dot{A}_{j}(\tau)\right\|: j \in \bar{q} \cup\{0\}\right) \leq \alpha,
\end{aligned}
$$

where $\delta(0)$ is a Dirac impulse at $t=0$. Note that Assumptions 2.3 imply $\left\|\sum_{j=0}^{q} A_{j}(t+\tau)\right\|-$ $\left\|\sum_{j=0}^{q} A_{j}(t)\right\| \mid \leq \mu T+v(t+\tau)$ for all $\tau \in[0, T]$, with the function $v: \mathbf{R}_{0+} \rightarrow \mathbf{R}_{0+}$ satisfying $v(t+\tau) \leq \alpha_{0} j+\alpha_{1 j}(t+\tau) \leq \alpha$ with $\alpha_{1 j}(t+\tau)=0$ if $\dot{A}_{j}(t+\tau)$ exists with $\left\|\dot{A}_{j}(t+\tau)\right\| \leq \alpha_{j}$ or $\alpha_{j}-\alpha_{0 j} \geq \alpha_{1 j}(t+\tau) \geq\left\|\Delta_{j}(t+\tau)\right\|$ if $\dot{A}_{j}(t+\tau)=\Delta_{j}(t+\tau) \delta(0)$, that is, at least one of its entries is impulsive.

Note that Assumption 2.1 implies that switching does not happen arbitrarily fast neither to reset parameters nor to reset-free ones. The subsequent result is direct.

Assertions 2.11. The following properties are true irrespective of the switching function:

(i) $t \notin \mathrm{ST}(\sigma) \Leftrightarrow \sigma\left(t^{+}\right)=\sigma(t)=0_{n \times(q+1) n}$ (i.e., a zero $n \times(q+1) n$-matrix);

(ii) $t \in \mathrm{ST}(\sigma) \Leftrightarrow \sigma\left(t^{+}\right)-\sigma(t) \neq 0_{n \times(q+1) n}$;

(iii) $t \notin \mathrm{ST}(\sigma) \Leftrightarrow \mathrm{ST}\left(\sigma, t^{+}\right)=\mathrm{ST}(\sigma, t)$;

(iv) $t \in \mathrm{ST}(\sigma) \Leftrightarrow \mathrm{ST}\left(\sigma, t^{+}\right) \neq \mathrm{ST}(\sigma, t)$.

Proof. (i) $\sigma\left(t^{+}\right)=\sigma(t)=0_{n \times(q+1) n} \Rightarrow t \notin \mathrm{ST}_{r}(\sigma) \wedge t \notin \mathrm{ST}_{\mathrm{rf}}(\sigma) \Leftrightarrow t \notin \mathrm{ST}(\sigma), t \notin \mathrm{ST} \Leftrightarrow \sigma(t)=0$ (since switching is not arbitrarily fast),

$$
\wedge\left(\sigma\left(t^{+}\right)-\sigma(t)=0_{n \times(q+1) n}\right) \Longleftrightarrow \sigma\left(t^{+}\right)=\sigma(t)=0_{n \times(q+1) n} .
$$

Property (i) has been proven. Property (ii) is the contrapositive logic proposition to Property (i), and thus equivalent, since switching is not arbitrarily fast.

Properties (iii)-(iv) are also contrapositive logic propositions, then equivalent since

$$
\begin{aligned}
\mathrm{ST}(\sigma, t):=\left\{t_{i} \in \mathrm{ST}(\sigma): t_{i}<t\right\} & \Longleftrightarrow \mathrm{ST}\left(\sigma, t^{+}\right):=\left\{t_{i} \in \mathrm{ST}(\sigma): t_{i}<t^{+}\right\}=\left\{t_{i} \in \mathrm{ST}(\sigma): t_{i} \leq t\right\} \\
& \Longleftrightarrow \mathrm{ST}\left(\sigma, t^{+}\right)\left\{\begin{array}{l}
=\mathrm{ST}(\sigma, t) \quad \text { if } t \notin \mathrm{ST}(\sigma), \\
\neq \mathrm{ST}(\sigma, t) \quad \text { if } t \in \mathrm{ST}(\sigma)
\end{array}\right.
\end{aligned}
$$

since switching cannot happen arbitrarily fast. Properties (iii)-(iv) have been proven. 
The subsequent global stability result is proven in Appendix A by guaranteeing that the Krasovsky-Lyapunov functional candidate below is indeed a Krasovsky-Lyapunov functional:

$$
V\left(t, x_{t}\right)=x^{T}(t) P(t) x(t)+\sum_{i=1}^{q} \sum_{j=0}^{q} \int_{-h_{i}-h_{j}}^{-h_{j}} \int_{t+\theta}^{t} x^{T}(\tau) S_{i j} x(\tau) d \tau d \theta
$$

Theorem 2.12. The following properties hold.

(i) Assume the following.

(i.a) The matrix functions $A_{j}(t)$, for all $j \in \bar{q} \cup\{0\}$ are subject to Assumption 2.1.

(i.b) The switching law $\sigma$ is such that

$$
Q(t):=\left[\begin{array}{ccccc}
H 1 & \widehat{h}_{1} P(t) A_{1}(t) M(t) & \cdots & \widehat{h}_{q} P(t) A_{q}(t) M(t) \\
\widehat{h}_{1} M^{T}(t) A_{1}^{T}(t) P(t) & -R_{1} & 0 \cdots & 0 \\
\vdots & 0 & \ddots & \vdots \\
\widehat{h}_{q} M^{T}(t) A_{q}^{T}(t) P(t) & 0 & \cdots & -R_{q}
\end{array}\right]<0,
$$

where H1 denotes $\left(\sum_{j=0}^{q} A_{j}^{T}(t)\right) P(t)+P(t)\left(\sum_{j=0}^{q} A_{j}(t)\right)+\dot{P}(t)+\sum_{i=1}^{q} \sum_{j=0}^{q} \widehat{h_{i}} S_{i j}$, for some time-differentiable real symmetric positive definite matrix function $P: \mathbf{R}_{0+} \rightarrow$ $\mathbf{R}^{n \times n}$ and some real symmetric positive definite matrices $S_{i j} \in \mathbf{R}^{n \times n}(\forall i \in \bar{q}, \forall j \in$ $\bar{q} \cup\{0\})$, where

$$
\begin{gathered}
M(t):=\left[A_{0}(t), \ldots, A_{q}(t)\right] \in \mathbf{R}^{n \times(q+1) n}, \\
R_{i}:=\operatorname{diag}\left(S_{i 0}, S_{i 1}, \ldots, S_{i q}\right) \in \mathbf{R}^{(q+1) n \times(q+1) n} ; \quad \forall i \in q, \quad \forall t \in \mathbf{R}_{0+} .
\end{gathered}
$$

Thus, the system (2.1) is globally asymptotically Lyapunov's stable for all delays $h_{i} \in$ $\left[0, \widehat{h}_{i}\right]$, for all $i \in \bar{q}$. A necessary condition is $\left(\sum_{j=0}^{q} A_{j}^{T}(t)\right) P(t)+P(t)\left(\sum_{j=0}^{q} A_{j}(t)\right)+$ $\dot{P}(t)<0$, for all $t \in \mathbf{R}_{0+}$ what implies that $\left(\sum_{j=0}^{q} A_{j}(t)\right)$ is a stability matrix of prescribed stability abscissa on $\mathbf{R}_{0+}$ except eventually on a real subinterval of finite measure of $\mathbf{R}_{0+}$.

(ii) Assume the following

(ii.a) $A_{i}(t)=A_{i j}$, for all $i \in \bar{q} \cup\{0\}$, for all $t \in \mathbf{R}_{0+}$ for some $j \in \bar{p}$ (eventually being dependent on $t$ ) satisfying Assumption 2.4.

(ii.b) The switching law $\sigma$ is such that $\mathrm{ST}_{r f}(\sigma)=\varnothing$ (i.e., it generates reset switching instants only) with $\mathrm{ST}_{r}(\sigma)$ being arbitrary, namely, the set of reset times is either any arbitrary strictly increasing sequence of nonnegative real values (i.e., the resetting switching never ends) or any finite set of strictly ordered nonnegative real numbers with a finite maximal (i.e., the resetting switching process ends in finite time). 
(ii.c)

$$
Q_{i}^{*}:=\left[\begin{array}{cccc}
\left(\sum_{j=0}^{q} A_{j i}^{T}\right) P^{*}+P^{*}\left(\sum_{j=0}^{q} A_{j i}\right)+\sum_{i=1}^{q} \sum_{j=0}^{q} \widehat{h}_{i} S_{i j}^{*} & \widehat{h}_{1} P^{*} A_{1 i} M_{1}^{*} & \cdots & \widehat{h}_{q} P^{*} A_{q i} M_{q}^{*} \\
\widehat{h}_{1} M_{1}^{* T} A_{1 i}^{T} P^{*} & -R_{1}^{*} & 0 \cdots & 0 \\
\vdots & 0 & \ddots & \vdots \\
\widehat{h}_{q} M_{q}^{* T} A_{q i}^{T} P^{*} & 0 & \cdots & -R_{q}^{*}
\end{array}\right]_{\forall i \in \bar{p}}
$$

for some $\mathbf{R}^{n \times n} \ni P^{*}=P^{* T}>0, \mathbf{R}^{n \times n} \ni S_{i j}^{*}=S_{i j}^{* T}>0(\forall i \in \bar{q}, \forall j \in \bar{q} \cup\{0\})$, where

$$
\begin{gathered}
M_{i}:=\left[A_{0 i}, \ldots, A_{q i}\right] \in \mathbf{R}^{n \times(q+1) n}, \\
R_{i}^{*}:=\operatorname{diag}\left(S_{i 0}^{*}, S_{i 1}^{*}, \ldots,\right) \in \mathbf{R}^{(q+1) n \times(q+1) n} ; \quad \forall i \in \bar{q}
\end{gathered}
$$

Thus, the switched system (2.1), obtained from switches among resetting systems (2.4), is globally asymptotically Lyapunov's stable and also globally exponentially stable for all delays $h_{i} \in\left[0, \widehat{h}_{i}\right]$, for all $i \in \bar{q}$. If (2.9) is replaced with $Q_{i}^{*} \leq$ $-2 \varepsilon I_{(q+1) n}<0$, for all $i \in \bar{q}$, and some $\varepsilon \in \mathbf{R}_{+}$then the state trajectory decays exponentially with rate $(-\varepsilon)<0$.

(iii) There is a sufficiently small $\widehat{h}:=\max _{i \in \bar{q}} \widehat{h}_{i}$ such that Property (i) holds for any $h_{i} \in\left[0, \widehat{h}_{i}\right]$, for all $i \in \bar{q}$ provided that all the delay-free resetting systems $(2.4) \dot{z}_{j}(t)=\left(\sum_{i=0}^{q} A_{i j}\right) z_{j}(t)$ fulfil Assumption 2.4, that is, they are globally exponentially stable.

It is of interest to discuss particular cases easy to test, guaranteeing Theorem 2.12 (i) .

\subsection{Sufficiency type asymptotic stability conditions obtained for constant symmetric matrices $P$ and $S_{i j}$}

Assume real constant symmetric matrices $P(t)=P$ and $S_{i j}$, for all $i \in \bar{q}$, for all $j \in \bar{q} \cup\{0\}$, for all $t \in \mathbf{R}_{0+}$ so that

$$
Q(t)=\left[\begin{array}{cccc}
H 2 & \widehat{h}_{1} P A_{1}(t) M(t) & \cdots & \widehat{h}_{q} P A_{q}(t) M(t) \\
\widehat{h}_{1} M^{T}(t) A_{1}^{T}(t) P & -R_{1} & 0 \cdots & 0 \\
\vdots & 0 & \ddots & \vdots \\
\widehat{h}_{q} M^{T}(t) A_{q}^{T}(t) P & 0 & \cdots & -R_{q}
\end{array}\right]<0,
$$

where $H 2$ denotes $\left(\sum_{j=0}^{q} A_{j}^{T}(t)\right) P+P\left(\sum_{j=0}^{q} A_{j}(t)\right)+\sum_{i=1}^{q} \sum_{j=0}^{q} \widehat{h}_{i} S_{i j}$. In this case, the KrasovskyLyapunov functional used in the proof of Theorem 2.12(i) holds defined with constant 
matrices for all time irrespective of being a switching-free instant or any switching instant (independently of its nature: reset time or reset-free switching instant). A practical test for (2.13) to hold follows. Consider $A_{i}^{*}(i \in \bar{q} \cup\{0\})$ such that the time invariant system (2.1) defined with $A_{i}(t) \rightarrow A_{i}^{*}$ is globally asymptotically Lyapunov's stable and define a stability real $n$-matrix $A^{*}:=\sum_{i=0}^{q} A_{i}^{*}$. Decompose $Q(t)=Q^{*}+\widetilde{Q}(t)$, where

$$
\begin{gathered}
Q^{*}:=\left[\begin{array}{cccc}
A^{* T} P+P A^{*}+\sum_{i=1}^{q} \sum_{j=0}^{q} \widehat{h}_{i} S_{i j} & \widehat{h}_{1} P A_{1}^{*} M^{*} & \ldots & \widehat{h}_{q} P A_{q}^{*} M^{*} \\
\widehat{h}_{1} M^{* T} A_{1}^{* T} P & -R_{1}^{*} & 0 \cdots & 0 \\
\vdots & 0 & \ddots & \vdots \\
\widehat{h}_{q} M^{* T} A_{q}^{* T} P & \vdots & & \\
& 0 & \cdots & -R_{q}^{*}
\end{array}\right], \\
\widetilde{Q}(t):=\left[\begin{array}{cccc}
\tilde{A}^{T}(t) P+P \tilde{A}(t) & \widehat{h}_{1} P \widetilde{\Delta}_{1}(t) & \cdots & \widehat{h}_{q} P \widetilde{\Delta}_{q}(t) \\
\widehat{h}_{1} \tilde{\Delta}_{1}^{T}(t) P & 0 & 0 \cdots & 0 \\
\vdots & 0 & \ddots & \vdots \\
\widehat{h}_{q} \tilde{\Delta}_{q}^{T}(t) P & 0 & \cdots & 0
\end{array}\right],
\end{gathered}
$$

where

$$
\begin{gathered}
M^{*}:=\operatorname{diag}\left(A_{0}^{*}, A_{1}^{*}, \ldots, A_{q}^{*}\right), \quad R_{i}^{*}:=\operatorname{diag}\left(S_{i 0}^{*}, S_{i 1}^{*}, \ldots, S_{i q}^{*}\right), \quad \widetilde{M}(t):=M(t)-M^{*}, \\
\tilde{A}_{i}(t):=A_{i}(t)-A^{*}, \quad \widetilde{A}(t):=\left(\sum_{j=0}^{q} A_{j i}(t)\right)-A^{*}, \\
\widetilde{\Delta}_{i}(t):=\left(\widetilde{A}_{i}(t) M^{*}+A_{i}^{*} \widetilde{M}(t)+\widetilde{A}_{i}(t) \stackrel{\leftrightarrow}{M}(t)\right) .
\end{gathered}
$$

If $t \in \mathrm{ST}(\sigma)$, then

$$
\begin{aligned}
\delta \widetilde{Q}(t) & :=\tilde{Q}\left(t^{+}\right)-\widetilde{Q}(t) \\
& =\left[\begin{array}{cccc}
H 3 & \widehat{h}_{1} P\left(\widetilde{\Delta}_{1}\left(t^{+}\right)-\widetilde{\Delta}_{1}(t)\right) & \cdots & \widehat{h}_{q} P\left(\widetilde{\Delta}_{1}\left(t^{+}\right)-\widetilde{\Delta}_{1}(t)\right) \\
\widehat{h}_{1}\left(\widetilde{\Delta}_{1}\left(t^{+}\right)-\widetilde{\Delta}_{1}(t)\right)^{T} P & 0 & 0 & 0 \\
\vdots & 0 & \ddots & \vdots \\
\widehat{h}_{q}\left(\widetilde{\Delta}_{1}\left(t^{+}\right)-\widetilde{\Delta}_{1}(t)\right)^{T} P & \vdots & & 0
\end{array}\right],
\end{aligned}
$$




$$
\begin{aligned}
\|\delta \widetilde{Q}(t)\|_{2} & \leq 2 \lambda_{\max }(P)\left[\left\|\tilde{A}^{T}\left(t^{+}\right)-\tilde{A}^{T}(t)\right\|_{2}+\sum_{i=1}^{q} \widehat{h}_{i}\left\|\widetilde{\Delta}_{i}\left(t^{+}\right)-\widetilde{\Delta}_{i}(t)\right\|_{2}\right] \\
& =2 \lambda_{\max }(P)\left[\left\|\sum_{i=0}^{q}\left(A_{i}^{T}\left(t^{+}\right)-A_{i}^{T}(t)\right)\right\|_{2}+\sum_{i=1}^{q} \widehat{h}_{i}\left\|\widetilde{\Delta}_{i}\left(t^{+}\right)-\widetilde{\Delta}_{i}(t)\right\|_{2}\right],
\end{aligned}
$$

where $H 3$ denotes $\left(\tilde{A}^{T}\left(t^{+}\right)-\widetilde{A}^{T}(t)\right) P+P\left(\left(\tilde{A}\left(t^{+}\right)-\tilde{A}(t)\right)\right)$, what leads to

$$
\begin{aligned}
\|\delta \widetilde{Q}(t)\|_{2} \leq 2 \lambda_{\max }(P)\left[(q+1) a^{+}+\widehat{h} q(\right. & a^{+}\left(\sum_{j=0}^{q}\left\|A_{j}^{*}\right\|_{2}+(q+1)\left\|A_{i}^{*}\right\|_{2}+(q+1)\left(a+a^{+}\right)\right) \\
& \left.\left.+(q+1)\left(2 a+a^{+}\right) a\right)\right]
\end{aligned}
$$

where $a:=\max _{i \in \bar{q} \cup\{0\}}\left(\left\|A_{i}\right\|_{2}\right)$ and $a^{+}:=\sup _{t \in \operatorname{ST}(\sigma)} \max _{i \in \bar{q} \cup\{0\}}\left(\left\|A_{i}\left(t^{+}\right)-A_{i}\right\|_{2}\right)$ (see (A.9) in Appendix A). Direct results from Theorem 2.12 which follow from (2.13) to (2.17) are given below.

Corollary 2.13. Consider in (2.9) replacements with constant real matrices $Q(t) \rightarrow Q^{*}=Q^{* T}$, $P(t) \rightarrow P=P^{T}>0, A_{i}(t) \rightarrow A_{i}^{*}, A(t) \rightarrow A^{*}:=\sum_{i=0}^{q} A_{i}^{*} ;$ for all $i \in \bar{q}$, for all $j \in \bar{q} \cup\{0\}$, for all $t \in \mathbf{R}_{0+}$ such that $A^{*}$ is a stability matrix. Then, Theorem 2.12(i) holds if $Q^{*}<0$ for any switching law $\sigma$ such that

(1) $\lambda_{\min }\left(-Q^{*}\right)=-\lambda_{\max }\left(Q^{*}\right)>\|\widetilde{Q}(t)\|_{2}:=\lambda_{\max }^{1 / 2}\left(\widetilde{Q}^{T}(t) \widetilde{Q}(t)\right)$, for all $t \in \mathbf{R}_{0+} \backslash \mathrm{ST}(\sigma)$,

(2) $a:=\max _{i \in \bar{q} \cup\{0\}}\left(\left\|A_{i}\right\|_{2}\right)$ and $a^{+}:=\sup _{t \in \mathrm{ST}(\sigma)} \max _{i \in \bar{q} \cup\{0\}}\left(\left\|A_{i}\left(t^{+}\right)-A_{i}\right\|_{2}\right)$ are sufficiently small such that

$$
\begin{aligned}
& \lambda_{\min }\left(-Q^{*}\right)-\|\widetilde{Q}(t)\|_{2} \\
&>2 \lambda_{\max }(P)\left[(q+1) a^{+}+\widehat{h} q\left(a^{+}\left(\sum_{j=0}^{q}\left\|A_{j}^{*}\right\|_{2}+(q+1) \max _{i \in \bar{q} \cup\{0\}}\left\|A_{i}^{*}\right\|_{2}+(q+1)\left(a+a^{+}\right)\right)\right.\right. \\
&\left.\left.+(q+1)\left(2 a+a^{+}\right) a\right)\right], \quad \forall t \in \operatorname{ST}(\sigma) .
\end{aligned}
$$

Corollary 2.14. Consider in (2.9) replacements with constant real matrices $Q(t) \rightarrow Q_{j}^{*}=Q_{j}^{* T}$, $P(t) \rightarrow P=P^{T}>0, A_{i j}(t) \rightarrow A_{i j}^{*}, A(t) \rightarrow A_{j}^{*}:=\sum_{i=0}^{q} A_{i j}^{*}$ for all $j \in \bar{p}$, for all $k \in \bar{q} \cup\{0\}$, for all $t \in \mathbf{R}_{0+}$ such that each $A_{j}^{*}(\forall j \in \bar{p})$ is a stability matrix with $A_{i j}^{*}$; for all $i \in \bar{q}$, for all $j \in \bar{p}$ being the parameterizations defining the resetting systems (2.4). Assume that the system (2.1) is one of the resetting systems (2.4) at $t=0$. Then, Theorem 2.12(i) holds with a common Krasovsky-Lyapunov function for all those resetting systems if $Q_{j}^{*}<0(\forall j \in \bar{p})$ for any switching law $\sigma$ such that 
(1) $\lambda_{\min }\left(-Q^{*}\right)=-\lambda_{\max }\left(Q^{*}\right)>\|\widetilde{Q}(t)\|_{2}:=\lambda_{\max }^{1 / 2}\left(\widetilde{Q}^{T}(t) \widetilde{Q}(t)\right)$, for all $t \in \mathbf{R}_{0+} \backslash \mathrm{ST}(\sigma)$,

(2) $a_{j}:=\max _{i \in \bar{q} \cup\{0\}}\left(\left\|A_{j i}\right\|_{2}\right)$ and $a_{j}^{+}:=\sup _{t \in \mathrm{ST}(\sigma)} \max _{i \in \bar{q} \cup\{0\}}\left(\left\|A_{i}\left(t^{+}\right)-A_{j i}\right\|_{2}\right)$ are sufficiently small such that

$$
\begin{aligned}
& \lambda_{\min }\left(-Q^{*}\right)-\|\widetilde{Q}(t)\|_{2} \\
&>2 \lambda_{\max }(P)\left[(q+1) a_{j}^{+}+\widehat{h} q\left(a_{j}^{+}\left(\sum_{i=0}^{q}\left\|A_{j i}\right\|_{2}+(q+1) \max _{i \in \bar{q} \cup\{0\}}\left\|A_{j i}\right\|_{2}\right)\right.\right. \\
&\left.\left.+(q+1)\left(a_{j}+a_{j}^{+}\right)+(q+1)\left(2 a_{j}+a_{j}^{+}\right) a_{j}^{+}\right)\right]
\end{aligned}
$$

$t \in \mathrm{ST}_{f r}(\sigma)$, provided that at time max $\left(t^{\prime}<t: t^{\prime} \in \mathrm{ST}_{r}(\sigma)\right)$, the system (2.1) coincides with at the $j \in \bar{p} r$ setting system (2.4).

The proof of Corollary 2.14 is close to that of Corollary 2.13 from (A.9) in Appendix A with the replacements $a \rightarrow a_{j}, a^{+} \rightarrow a_{j}^{+}$for all $j \in \bar{p}$. If (2.19) is rewritten with the replacements $a_{j} \rightarrow \bar{a}:=\max _{j \in \bar{p}} a_{j}, a_{j}^{+} \rightarrow \vec{a}^{+}:=\max _{j \in \bar{p}} a_{j}^{+}$then the reformulated weaker Corollary 2.14 is valid for all $t \in \mathrm{ST}_{\mathrm{fr}}(\sigma)$ irrespective of the preceding reset switching. A result which guarantees Corollary 2.13, and then Theorem 2.12(i), is now obtained by replacing the $(1,1)$ block matrix of $Q^{*}$ by a Lyapunov matrix equality as follows. Consider a real $n$ matrix $Q_{0}^{*}=Q_{0}^{* T}>0$ such that $\lambda_{\min }\left(Q_{0}^{*}\right)>\lambda_{\max }\left(\sum_{i=1}^{q} \sum_{j=0}^{q} \widehat{h}_{i} S_{i j}\right)$ and $P:=\int_{0}^{\infty} e^{A^{* T} \tau} Q_{0}^{*} e^{A^{*} \tau} d \tau$ satisfying the Lyapunov equation $A^{* T} P+P A^{*}=-Q_{0}^{*}<0$ as its unique solution. Note that $\lambda_{\max }(P) \leq K^{*} \lambda_{\max }\left(Q_{0}^{*}\right) / 2 \rho^{*}$ for some $K^{*} \in \mathbf{R}_{+}$, where $-\rho^{*}<0$ is the stability abscissa of $A^{*}$ with $\left\|e^{A *^{T} t}\right\| \leq \sqrt{K^{*}} e^{-\rho^{*} t}$ for all $t \in \mathbf{R}$. Define the decomposition $Q(t)=\bar{Q}^{*}+\tilde{\bar{Q}}(t)$, where

$$
\begin{aligned}
& \bar{Q}^{*}:=\operatorname{Block} \operatorname{Diag}\left(-Q_{0}^{*}+\sum_{i=1}^{q} \sum_{j=0}^{q} \widehat{h}_{i} S_{i j},-R_{1}^{*}, \ldots,-R_{q}^{*}\right), \\
& \tilde{\bar{Q}}(t):=\left[\begin{array}{cccc}
\tilde{A}^{T}(t) P+P \widetilde{A}(t) & \widehat{h}_{1} P\left(A_{1}^{*} M^{*}+\widetilde{\Delta}_{1}(t)\right) & \cdots & \widehat{h}_{q} P\left(A_{q}^{*} M^{*}+\widetilde{\Delta}_{q}(t)\right) \\
\widehat{h}_{1}\left(A_{1}^{*} M^{*}+\widetilde{\Delta}_{1}(t)\right)^{T} P & 0 & 0 \cdots & 0 \\
\vdots & 0 & \ddots & \vdots \\
\widehat{h}_{q}\left(A_{q}^{*} M^{*}+\widetilde{\Delta}_{q}(t)\right)^{T} P & 0 & \cdots & 0
\end{array}\right] \\
& \Longrightarrow\|\tilde{\bar{Q}}(t)\|_{2} \leq\left(\frac{K^{*} \lambda_{\max }\left(Q_{0}^{*}\right)}{\rho^{*}}\right)\left(\left\|\tilde{A}^{T}(t)\right\|_{2}+\sum_{i=1}^{q} \widehat{h}_{i}\left\|A_{i}^{*} M^{*}+\widetilde{\Delta}_{i}(t)\right\|_{2}\right) .
\end{aligned}
$$

Thus, the subsequent result follows from Corollary 2.13 and (2.20). 
Corollary 2.15. Consider the matrices of Corollary 2.13 with $A^{*}$ being a stability matrix with stability abscissa $\left(-\rho^{*}\right)<0$ which satisfies the Lyapunov equation $A^{*^{T}} P+P A^{*}=-Q_{0}^{*}<0$. Then, Theorem 2.12(i) holds if $Q^{*}<0$ for any switching law $\sigma$ such that

$$
\begin{aligned}
& \lambda_{\min }\left(-\bar{Q}^{*}\right)=-\lambda_{\max }\left(\bar{Q}^{*}\right) \\
&>\left(\frac{K^{*} \lambda_{\max }\left(Q_{0}^{*}\right)}{\rho^{*}}\right)\left(\left\|\tilde{A}^{T}(t)\right\|_{2}+\sum_{i=1}^{q} \widehat{h}_{i}\left\|A_{i}^{*} M^{*}+\widetilde{\Delta}_{i}(t)\right\|_{2}\right), \\
& \forall t \in \mathbf{R}_{0+}, \quad \forall t \in \operatorname{ST}(\sigma) .
\end{aligned}
$$

\subsection{Sufficiency type asymptotic stability conditions obtained for time-varying symmetric matrices $P(t), S_{i j}(t)=S_{i j}$}

The following result, which is proven in Appendix A, holds.

Theorem 2.16. Under Assumptions 2.1-2.3, the following properties hold.

(i) The switched system (2.1) is globally asymptotically Lyapunov's for any delays $h_{i} \in\left[0, \widehat{h}_{i}\right]$ for all $i \in \bar{q}$ for some $\widehat{h}:=\max _{i \in \bar{q}}\left(\widehat{h}_{i}\right)$ and any switching law $\sigma$ such that

(a) the switching instants are arbitrary;

(b) $\max \left(\right.$ ess sup $\left.\left\|\dot{A}_{j}(t)\right\|: t \in \mathbf{R}_{0+}, j \in \bar{p}\right)$ is sufficiently small compared to the absolute value of the prescribed stability abscissa of $\sum_{j=0}^{q} A_{j}(t)$;

(c) the support testing matrix of distributional derivatives $\Gamma_{\text {Adj }}(t)$ of the same matrices are semidefinite negative for all time instants, where the conventional derivatives do not exist (i.e., $\dot{A}_{j}(t)=\Gamma_{\text {Adj }}(t)(t) \delta(0)$ ).

(ii) The switched system (2.1) is globally exponentially stable for any delays $h_{i} \in\left[0, \widehat{h}_{i}\right]$ for all $i \in \bar{q}$ for some $\widehat{h}:=\max _{i \in \bar{q}}\left(\widehat{h}_{i}\right)$ such that

(a) $\max \left(\operatorname{ess} \sup \left\|\dot{A}_{j}(t)\right\|: t \in \mathbf{R}_{0+}, j \in \bar{p}\right)$ is sufficiently small compared to the absolute value of the prescribed stability abscissa of $\sum_{j=0}^{q} A_{j}(t)$;

(b) $\max \left(\left\|\Gamma_{\text {Adj }}(t)(t)\right\|: \dot{A}_{j}(t)=\Gamma_{\text {Adj }}(t)(t) \delta(0), \forall t \in \mathrm{ST}(\sigma), j \in \bar{p}\right)$ is sufficiently small compared to the timeintervals in between any two consecutive switching instants.

Furthermore, if Assumptions 2.1-2.4 hold, then

(iii) the switched system (2.1) is globally exponentially stable for any delays $h_{i} \in\left[0, \widehat{h}_{i}\right]$ for all $i \in \bar{q}$, for some $\widehat{h}:=\max _{i \in \bar{q}}\left(\widehat{h}_{i}\right)$ such that

(a) $\max \left(\right.$ ess sup $\left.\left\|\dot{A}_{j}(t)\right\|: t \in \mathbf{R}_{0+}, j \in \bar{p}\right)$ is sufficiently small compared to the absolute value of the prescribed stability abscissa of $\sum_{j=0}^{q} A_{j}(t)$; 
(b) the switching law $\sigma$ is such that

(a) $\max \left(\left\|\Gamma_{\text {Adj }}(t)\right\|: \dot{A}_{j}(t)=\Gamma_{\text {Adj }}(t) \delta(0), \forall t \in \mathrm{ST}_{r f}(\sigma), j \in \bar{p}\right)$ is sufficiently small compared to the lengths of time intervals between any two consecutive switching instants;

(b) it exists a common Krasovsky-Lyapunov functional $V\left(t, x_{t}\right)$ defined with constant matrices $\bar{P}=\bar{P}^{T}>0$ and $\bar{S}_{i j}=\bar{S}_{i j}^{T}>0$, for all $(i, j) \in(\bar{q} \cup\{0\}) \times \bar{q}$ for all the time-invariant resetting systems (2.4) and some of the subsequent conditions hold for all $t \in \mathrm{ST}_{r}(\sigma)$ under the resetting action $P\left(t^{+}\right)=\bar{P}$; for all $(i, j) \in$ $(\bar{q} \cup\{0\}) \times \bar{q}:$

(b.1) $V\left(t^{+}, x_{t}\right) \leq V\left(t, x_{t}\right)$ which is guaranteed, in particular, if $P\left(t^{+}\right)=\bar{P} \leq P(t)$,

(b.2) the tradeoff $(a)$ is respected between sufficiently small norms of the matrices of distributional derivatives and the length $\left|t-t^{\prime}\right|$, at any $t \in \mathrm{ST}_{r}(\sigma)$, if any, where the condition (b.1) is not satisfied, where $t^{\prime}=\max \left(\tau \in \mathbf{R}_{0+}: \operatorname{ST}(\sigma) \ni\right.$ $\tau<t)$.

The characterization of the "sufficient smallness" of the involved magnitudes in Theorem 2.16 is given explicitly in its proof. The proof considers that when some entry time derivative of the involved matrices does not exist, it equivalently exists a distributional derivative at this time instant which is equivalent to the existence of a bounded jump-type discontinuity in its integral, so that the corresponding time instant is in fact a switching instant. The sufficiently large time intervals required in between any two consecutive switching times compared with the amplitudes of the amplitude (in terms of norm errors) among consecutive parameterizations are related to the need for a minimum residence time at each parameterization for the case when those ones do not possess a common KrasovskyLyapunov functional.

\section{Asymptotic stability independent of the delays}

Some results concerning sufficiency type properties of global asymptotic stability independent of the delays, that is, for any $h_{i} \in \mathbf{R}_{0+}$, for all $i \in \bar{q}$ of the switched system (2.1) are obtained under very close guidelines as those involved in the results on stability dependent of the delays given in Section 2. The Krasovsky-Lyapunov functional candidate of Section 2 and Appendix A is modified as follows:

$$
V\left(t, x_{t}\right)=x^{T}(t) P(t) x(t)+\sum_{i=1}^{q} \int_{t-h_{i}}^{t} x^{T}(\tau) S_{i}(\tau) x(\tau) d \tau
$$

whose time derivative along the state-trajectory solution of (2.1) is

$$
\begin{aligned}
\dot{V}\left(t, x_{t}\right)= & x^{T}(t)\left(A^{T}(t) P(t)+P(t) A(t)+\sum_{i=1}^{q} S_{i}(t)+\dot{P}(t)\right) x(t) \\
& +2 x^{T}(t) \sum_{i=1}^{q} P A_{i}(t) x\left(t-h_{i}\right)-\sum_{i=1}^{q} x^{T}\left(t-h_{i}\right) S_{i}\left(t-h_{i}\right) x\left(t-h_{i}\right) \\
= & \bar{x}^{T}(t) Q^{\prime}(t) \bar{x}(t)<0
\end{aligned}
$$


for all nonzero $\bar{x}^{T}(t)=\left(x^{T}(t), x^{T}\left(t-h_{1}\right), \ldots, x^{T}\left(t-h_{q}\right)\right)$ if

$$
Q^{\prime}(t):=\left[\begin{array}{cccc}
A^{T}(t) P(t)+P(t) A(t)+\sum_{i=1}^{q} S_{i}(t)+\dot{P}(t) & P A_{1}(t) & \cdots & P A q(t) \\
A_{1}^{T}(t) P & -S_{1}\left(t-h_{1}\right) & 0 & \cdots \\
\vdots & & \vdots & 0 \\
A_{q}^{T}(t) P & 0 & -S_{q}\left(t-h_{q}\right)
\end{array}\right]<0 .
$$

Assumption 2.1 of Section 2 remains unchanged while Assumptions 2.2 and 2.4 of Section 2 are modified under similar justifications as follows.

Assumption 3.1. All the eigenvalues $\lambda_{i}\left(A_{0}(t)\right)$ of the matrix function $A_{0}(t)$ satisfy $\operatorname{Re} \lambda_{i}\left(A_{0}(t)\right) \leq-\rho_{00}<0$; for all $t \in \mathbf{R}_{0+}$, for all $i \in \bar{\sigma}(\sigma \leq n)$ for some $\rho_{00} \in \mathbf{R}_{+}:=\{0<z \in \mathbf{R}\}$; that is, $A_{0}(t)$ is a stability matrix, for all $t \in \mathbf{R}_{0+}$.

Assumptions 3.2. $A_{j}: \mathbf{R}_{0+} \rightarrow \mathbf{R}^{n \times n}$ are almost everywhere time-differentiable with essentially bounded time derivative, for all $j \in \bar{q} \cup\{0\}$ possessing eventual isolated bounded discontinuities, then ess $\sup _{t \in \mathbf{R}_{0+}}\left(\left\|\dot{A}_{0}(t)\right\|\right) \leq \gamma_{0 \ell}<\infty$ with $\gamma_{0 \ell}$ being a $\ell$-norm dependent nonnegative real constant and, furthermore, $\int_{t}^{t+T}\left\|\dot{A}_{0}(\tau)\right\| d \tau \leq \mu_{0} T+\alpha_{0}$ for some $\alpha_{0}, \mu_{0} \in$ $\mathbf{R}_{+}$, for all $t \in \mathbf{R}_{0_{+}}$, and some fixed $T \in \mathbf{R}_{0+}$ independent of $t$. If the time derivative does not exist then it is defined in the distributional sense as in Assumptions 2.3.

Assumption 3.3 (for the resetting systems). All the eigenvalues $\lambda_{k}\left(A_{0 j}\right)$ satisfy $\operatorname{Re} \lambda_{k}\left(A_{j 0}\right) \leq$ $-\rho_{00}<0$; for all $t \in \mathbf{R}_{0+}$, for all $k \in \bar{\sigma}_{0 j}\left(\sigma_{0 j} \leq n\right)$, for all $j \in \bar{p}$; that is, $A_{0 j}$ are constant stability matrices with prescribed stability abscissa.

A parallel result to Theorem 2.12(i)-(ii) is the following.

Theorem 3.4. The subsequent properties hold.

(i) Assume that

(i.a) the matrix functions $A_{j}(t)$, for all $j \in \bar{q} \cup\{0\}$ are subject to Assumption 2.1;

(i.b) the switching law $\sigma$ is such that $Q^{\prime}(t)<0$, (3.3), for all $t \in \mathbf{R}_{0+}$

for some time-differentiable real symmetric positive definite matrix function $P: \mathbf{R}_{0+} \rightarrow$ $\mathbf{R}^{n \times n}$ and some real symmetric positive definite matrix functions $S_{i}: \mathbf{R}_{0+} \rightarrow \mathbf{R}^{n \times n}(\forall i \in$ $\bar{q})$. Thus, the system (2.1) is globally asymptotically Lyapunov's stable independent of the delays (i.e., for all delays $h_{i} \in[0, \infty)$, for all $i \in \bar{q}$ ). A necessary condition is $A_{0}^{T}(t) P(t)+$ $P(t) A_{0}(t)+\dot{P}(t)<0$, for all $t \in \mathbf{R}_{0+}$ what implies that $A_{0}(t)$ is a stability matrix of prescribed stability abscissa on $\mathbf{R}_{0+}$ except eventually on a real subinterval of finite measure of $\mathbf{R}_{0+}$.

(ii) Assume that

(ii.a) $A_{j}(t)=A_{j i}$, for all $j \in \bar{q} \cup\{0\}$, for all $t \in \mathbf{R}_{0+}$ for some $i \in \bar{p}$ (eventually being dependent on t) satisfying Assumption 3.3; 
(ii.b) the switching law $\sigma$ is such that $\operatorname{ST}_{r f}(\sigma)=\varnothing$ (i.e., it generates reset switching instants only) with $\mathrm{ST}_{r}(\sigma)$ being arbitrary, namely, the set of reset times is either any arbitrary strictly increasing sequence of nonnegative real values (i.e., the resetting switching never ends) or any finite set of strictly ordered nonnegative real numbers with a finite maximal (i.e., the resetting switching ends in finite time);

(ii.c)

$$
Q_{i}^{* \prime}:=\left[\begin{array}{cccc}
A_{0 i}^{T} P^{*}+P^{*} A_{0 i}+\sum_{i=1}^{q} S_{i}^{*} & P^{*} A_{1 i} & \cdots & P^{*} A_{q i} \\
A_{1 i}^{T} P^{*} & -S_{1}^{*} & 0 \cdots & 0 \\
\vdots & 0 & \ddots & \vdots \\
A_{q i}^{T} P^{*} & \vdots & & \\
& 0 & \cdots & -S_{q}^{*}
\end{array}\right]<0 ; \quad \forall i \in \bar{p}
$$

for some $\mathbf{R}^{n \times n} \ni P^{*}=P^{* T}>0, \mathbf{R}^{n \times n} \ni S_{i}^{*}=S_{i}^{* T}>0(\forall i \in \bar{q})$. Thus, the switched system (2.1), obtained from switches among resetting systems (2.4), is globally asymptotically Lyapunov's stable and also globally exponentially stable independent of the delays for all $i \in \bar{q}$. If (3.4) is replaced with $Q_{i}^{* \prime} \leq-2 \varepsilon I_{(q+1) n}<0$, for all $i \in \bar{q}$, and some $\varepsilon \in \mathbf{R}_{+}$then the state trajectory decays exponentially with rate $(-\varepsilon)<0$.

Parallel results to Corollaries 2.13-2.15 are direct from Theorem 3.4 with the replacements $A_{0}(t) \rightarrow A_{0}^{*}$ (a constant stability matrix), $A_{0 j}(t) \rightarrow A_{0 j}^{*}$, for all $j \in \bar{p}$ (a set of constant stability matrices with prescribed stability abscissa for the resetting configurations). Also, the subsequent result for global asymptotic stability independent of the delays, which is close to Theorem 2.16, follows by replacing Assumptions 2.2-2.4 by Assumptions 3.1-3.3.

Theorem 3.5. Under Assumptions 2.1 and 3.1-3.2, the following properties hold.

(i) The switched system (2.1) is globally asymptotically Lyapunov's stable independent of the delays, that is, for any delays $h_{i} \in[0, \infty)$, for all $i \in \bar{p}$ and any switching law $\sigma$ such that

(a) the switching instants are arbitrary;

(b) $\max \left(\right.$ ess sup $\left.\left\|\dot{A}_{0}(t)\right\|: t \in \mathbf{R}_{0+}\right)$ is sufficiently small compared to the absolute value of the prescribed stability abscissa of $A_{0}(t)$;

(c) the support testing matrix of distributional derivatives $\Gamma_{\text {Ado }}(t)$ of the same matrices are semidefinite negative for all time instants, where the conventional derivatives do not exist (i.e., if $\left.\dot{A}_{0}(t)=\Gamma_{A d 0}(t)(t) \delta(0)\right)$.

(ii) The switched system (2.1) is globally exponentially stable independent of the delays if

(a) $\max \left(\operatorname{ess} \sup \left\|\dot{A}_{0}(t)\right\|: t \in \mathbf{R}_{0+}\right)$ is sufficiently small compared to the absolute value of the prescribed stability abscissa of $A_{0}(t)$;

(b) $\max \left(\left\|\Gamma_{A d 0}(t)(t)\right\|: \dot{A}_{0}(t)=\Gamma_{A d 0}(t)(t) \delta(0), \forall t \in \mathrm{ST}(\sigma)\right)$ is sufficiently small compared to the time intervals in between any two consecutive switching instants. 
If Assumptions 2.1 and 3.1-3.3 hold, then

(iii) the switched system (2.1) is globally exponentially stable independent of the delays if $\max \left(\right.$ ess sup $\left.\left\|\dot{A}_{0}(t)\right\|: t \in \mathbf{R}_{0_{+}}\right)$is sufficiently small compared to the absolute value of the prescribed stability abscissa of $A_{0}(t)$ and, furthermore, the switching law $\sigma$ is such that

(a) At reset-free switching instants, $\max \left(\left\|\Gamma_{A d 0}(t)(t)\right\|: \dot{A}_{0}(t)=\Gamma_{A d 0}(t)(t) \delta(0), \forall t \in\right.$ $\left.\mathrm{ST}_{r f}(\sigma)\right)$ is sufficiently small compared to the time intervals in between any two consecutive reset switching instants;

(b) there exists a common Krasovsky-Lyapunov functional $V\left(t, x_{t}\right)$ defined with constant matrices $P(t) \rightarrow \bar{P}=\bar{P}^{T}>0$ and $S_{i}(t) \rightarrow \bar{S}_{i}=\bar{S}_{i}^{T}>0$, for all $i \in \bar{q} \cup\{0\}$ in (3.1) for all the time-invariant resetting systems (2.4) and some of the conditions (b.1)(b.2) of Theorem 2.16 hold for all $t \in \mathrm{ST}_{r}(\sigma)$ under the resetting action $P\left(t^{+}\right)=\bar{P}$; for all $i \in \bar{q} \cup\{0\}$.

\section{Simulation examples and potential future research}

In this section, some simulation examples showing numerically the application of the results introduced below are carried out. The section contains two examples: one related to the delay-dependent stability property introduced in Section 2 and another concerning the delayindependent one considered in Section 3. The resetting systems and the remaining potential jumps in any parameters are considered without explicit separation of the two phenomena since such a separation is not relevant for stability properties.

\subsection{Delay-dependent stability}

Consider the delay system $\dot{x}(t)=A_{0}(t) x(t)+A_{1}(t) x(t-h)$, where $h=0.75$ second and each resetting system (2.4) with $p=2$ is defined by

$$
\begin{array}{ll}
A_{01}(t)=\left(\begin{array}{cc}
-1.3 & \frac{\cos t}{4} \\
0 & -2.5
\end{array}\right), & A_{11}(t)=\left(\begin{array}{cc}
-2.2 & a(t) \\
0 & -2.1
\end{array}\right), \\
A_{02}(t)=\left(\begin{array}{cc}
-1 & \frac{\sin t}{4} \\
0 & -2
\end{array}\right), & A_{12}(t)=\left(\begin{array}{cc}
-1.5 & b(t) \\
0 & -2
\end{array}\right)
\end{array}
$$

with $a(t)=1 /(\lfloor t\rfloor+t+1)$ and $b(t)=t /\left(\left[\left.t\right|^{2}+1\right)\right.$, where $\lfloor\cdot\rfloor$ denotes the largest integer not larger than $(\cdot)$ and $[\cdot]$ denotes the smallest integer not smaller than $(\cdot)$. Note that $a(t)$ and $b(t)$ are discontinuous functions at integer values of time. A graphical representation of these functions is shown in Figures 1 and 2.

Initially, it will be checked that Theorem 2.16(ii) conditions hold. Firstly, the switching instants between resetting systems have been selected arbitrarily and defined by Figure 3 .

Secondly, the time derivatives of the resetting systems defined above are given on each real interval $(k, k+1) \subset R$ with $k \in N$ by

$$
\dot{A}_{01}=\left(\begin{array}{cc}
0 & \frac{-\sin t}{4} \\
0 & 0
\end{array}\right), \quad \dot{A}_{11}=\left(\begin{array}{cc}
0 & \dot{a}(t) \\
0 & 0
\end{array}\right), \quad \dot{A}_{02}=\left(\begin{array}{cc}
0 & \frac{\cos t}{4} \\
0 & 0
\end{array}\right), \quad \dot{A}_{12}=\left(\begin{array}{cc}
0 & \dot{b}(t) \\
0 & 0
\end{array}\right)
$$




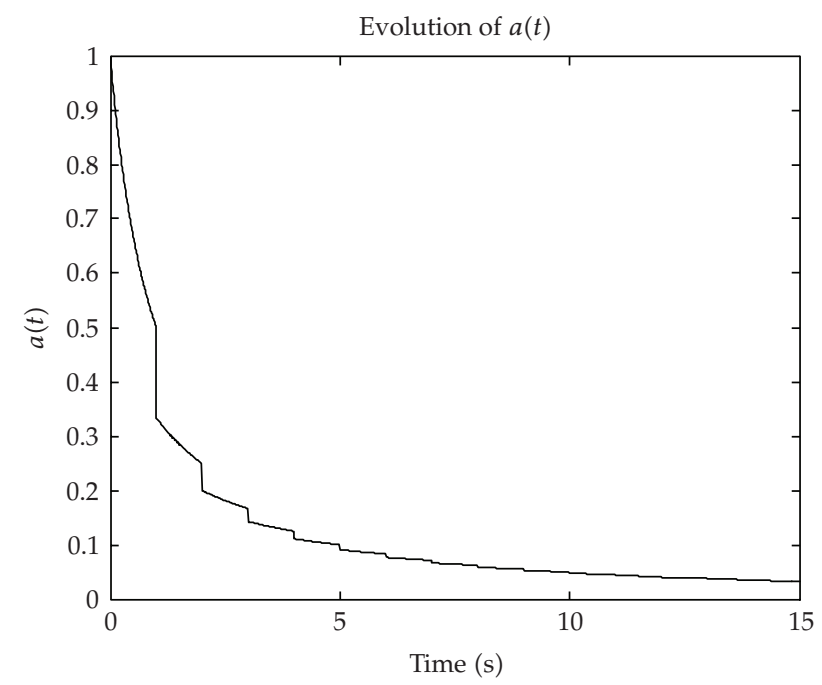

Figure 1: Graphical representation of $a(t)$.

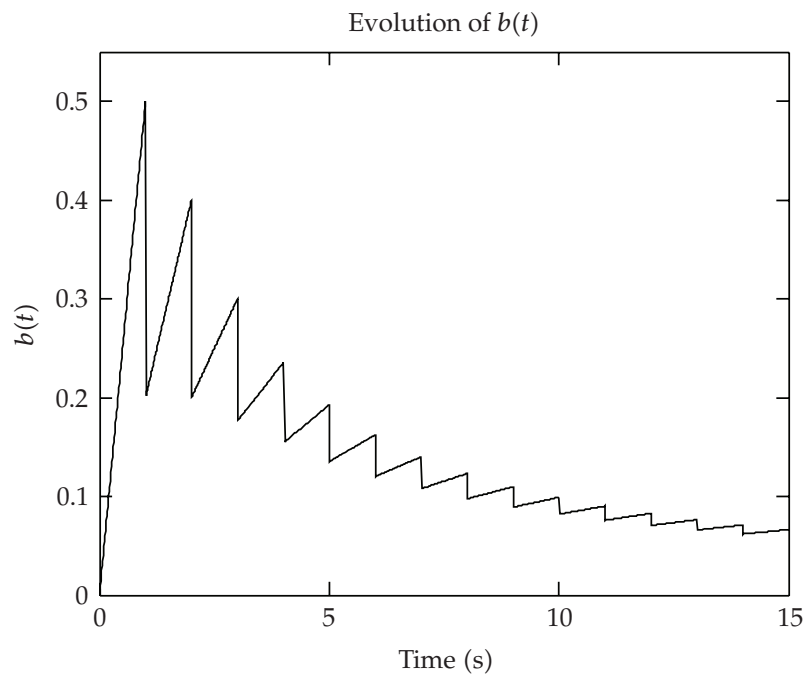

Figure 2: Graphical representation of $b(t)$.

with $\dot{a}(t)=-1 /(k+t+1) ; \dot{b}(t)=1 /\left((1+k)^{2}+1\right)$. Thus, the "sufficiently smallness condition" mentioned in Theorem 2.16(i) is fulfilled according to its proof in Appendix A if $2\left(\rho_{0} / K_{0}\right)^{2}>$

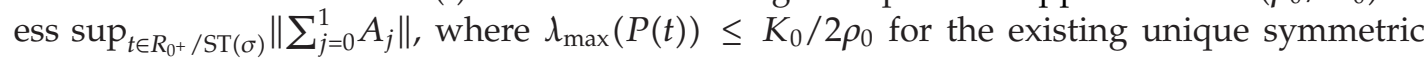
positive definite solution $P(t)$ of $\left(\sum_{j=0}^{1} A_{j}^{T}\right) P(t)+P(t)\left(\sum_{j=0}^{1} A_{j}\right)=-I_{n}$. Numerical computations lead to $10.33=2\left(\rho_{0} / K_{0}\right)^{2}>$ ess $\sup _{t \in R_{0^{+}} / \mathrm{ST}(\sigma)}\left\|\sum_{j=0}^{1} A_{j}\right\|=1.25$ which guarantees the fulfilment the second item of the theorem. Finally, from Figures 1 and 2 above it becomes apparent that the distributional derivative at integer time instants (where the ordinary derivative does not exist) is negative since $a(t)>a\left(t^{+}\right)$and $b(t)>b\left(t^{+}\right)$for all $t \in N$. Therefore, from Theorem 2.16(ii), the space-state trajectories of the solution asymptotically converge to zero as time evolves as Figure 4 shows. 


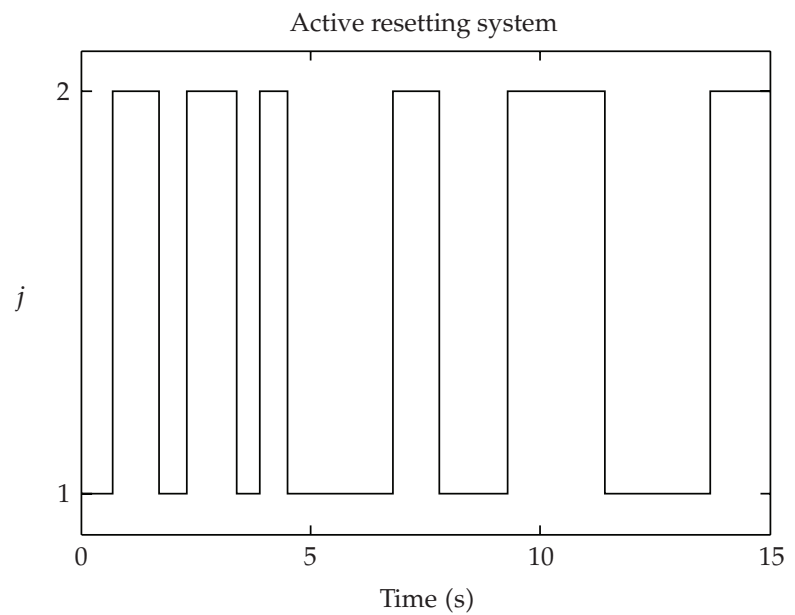

Figure 3: Sequence of switching instants between resetting systems.

The phase plane is shown in Figure 5, where it can be appreciated the convergence of the state evolution to the origin. Note that delay-dependent stability is achieved. As simulations show, the system becomes unstable as delay exceeds a certain threshold. Global asymptotic stability is guaranteed within the delay variation interval $[0,1]$.

\subsection{Delay-independent stability}

This example is devoted to the delay independent stability ensured by Theorem 3.5(i). Thus, consider the system $\dot{x}(t)=A_{0}(t) x(t)+A_{1}(t) x(t-h)$ and the resetting systems

$$
A_{01}=\left(\begin{array}{cc}
-1 & \frac{1}{2} \\
0 & -3
\end{array}\right), \quad A_{02}=\left(\begin{array}{cc}
-2.5 & 2 \\
0 & -1.6
\end{array}\right), \quad A_{1}=\left(\begin{array}{cc}
-2.5 & a(t) \\
0 & -1.6
\end{array}\right)
$$

with $a(t)=2+1 /(1+t)^{n / 10}$ and $n=\lfloor t\rfloor$. Again, this function possesses bounded discontinuities at integer values of time as Figure 6 shows.

Furthermore, the conditions of Theorem 3.5(i) are especially easy to verify since the resetting matrices $A_{0 i}$ are time-invariant and hence its time-derivatives are identically zero. The switching sequence is the same as depicted in Figure 3. Figures 7, 8, 9, and 10 show the convergence of the state trajectories of the system to zero for different values of the delay showing the delay independence property.

\subsection{Potential future research}

It is convenient to point out that the above ideas could be used for a better adjustment in Biology and Ecology mathematical models which have received increasing attention recently concerning epidemic propagation, species evolution, predation, and so forth (see, e.g., [25$35]$ ), which can also include delays to better fix the trajectory solutions. For instance, a control theory point of view is given in [29] for the standard Beverton-Holt equation in Ecology which has two parameterizing sequences, namely, the environment carrying capacity (related 


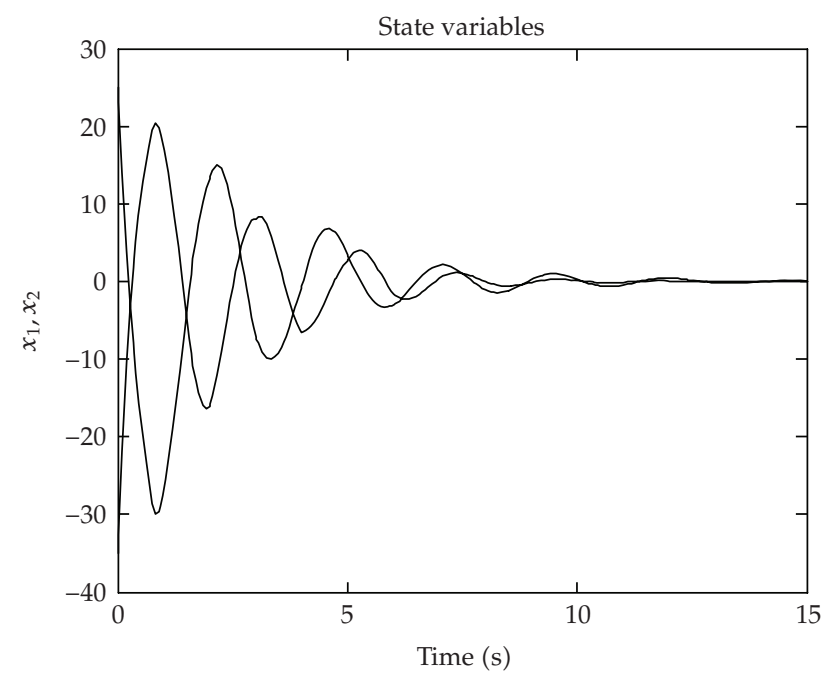

Figure 4: Convergence to zero of the state-trajectories.

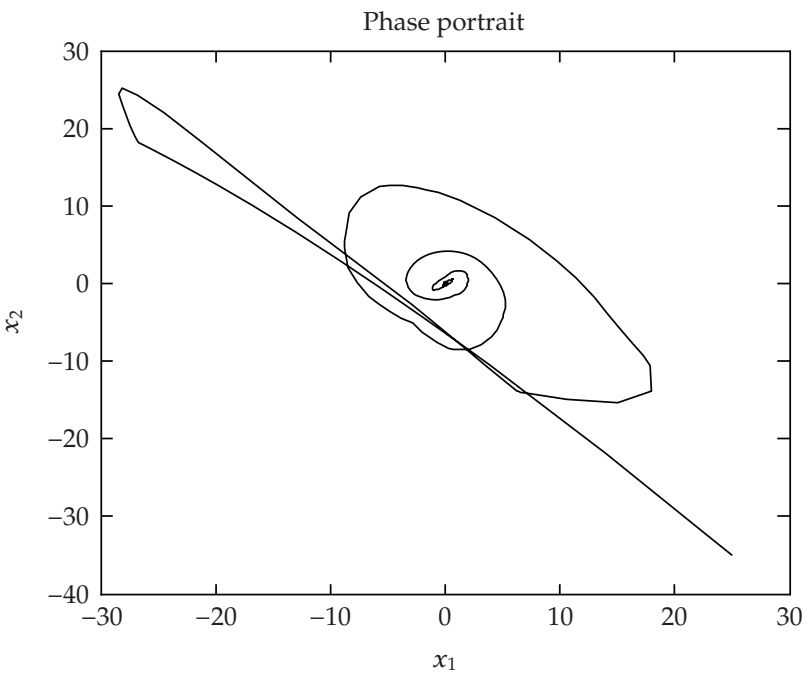

Figure 5: Phase portrait of the evolution of the system.

to a favorable or not habitat for the population) and the intrinsic growth rate (related to the population ability to grow). The inverse of the environment carrying capacity is the control variable. The objective is that the solution trajectory matches a prescribed reference one. The stability results and the matching properties are revisited in [30] for the generalized Beverton-Holt equation which possesses two extra parameterizing sequences, namely, the harvesting quota (related to human intervention like, fishing/hunting) and the independent consumption (related to perturbations in the population levels). The above two models are discrete with a one-step delay. Other control variables apart from the carrying capacity inverse are taken in [31] and comparative results with the former case are provided. Finally, a modified generalized Beverton-Holt is discussed in [32] which is a more complex model 


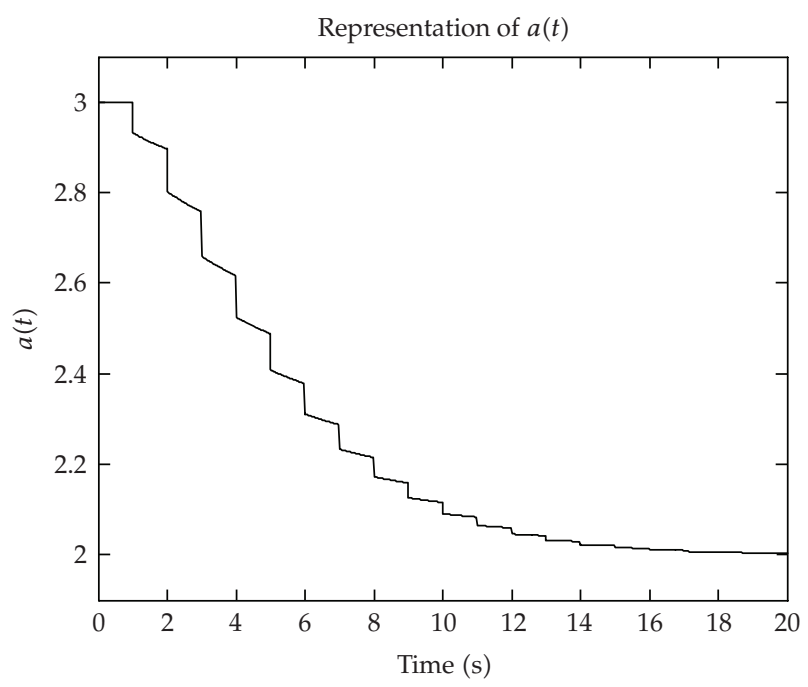

Figure 6: Graphical representation of $a(t)$.

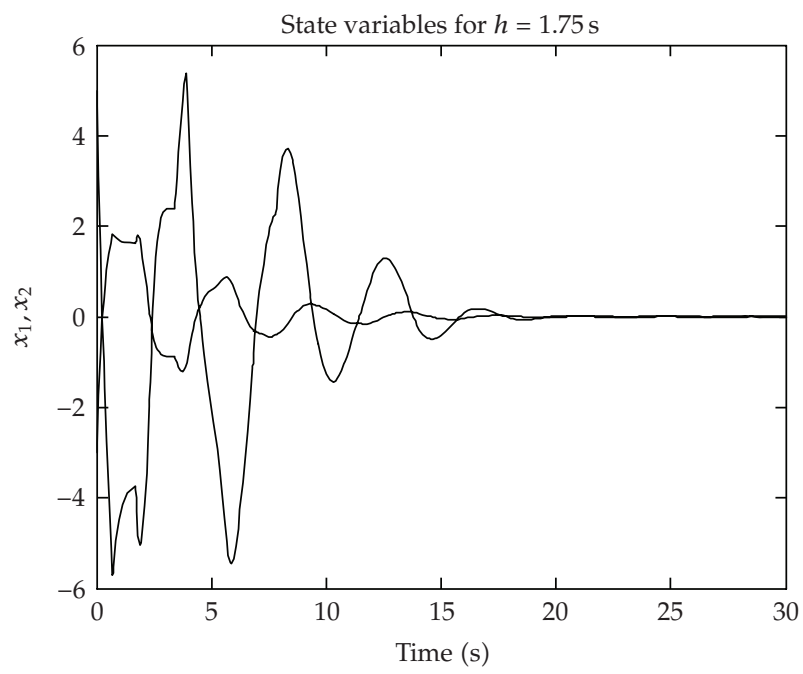

Figure 7: State trajectories evolution for a delay of 1.75 seconds.

than the former model in [30]. This model has a delay of two sampling periods, the new one introduces a penalty in the dynamics for large levels of populations. The strategy seems to be appropriate for certain populations of insects which have several reproduction cycles per year and whose population tends to blast in very short periods of time what makes it to fall after very much as a result. If a comparative is made between the various (standard, generalized, and modified generalized) models, one sees that the foreseen population evolution might depend significantly on the chosen model. Therefore, a switching model strategy between several kinds of single models each one subject to a set of distinct parameterizations could be useful to better adjust experimental data. 


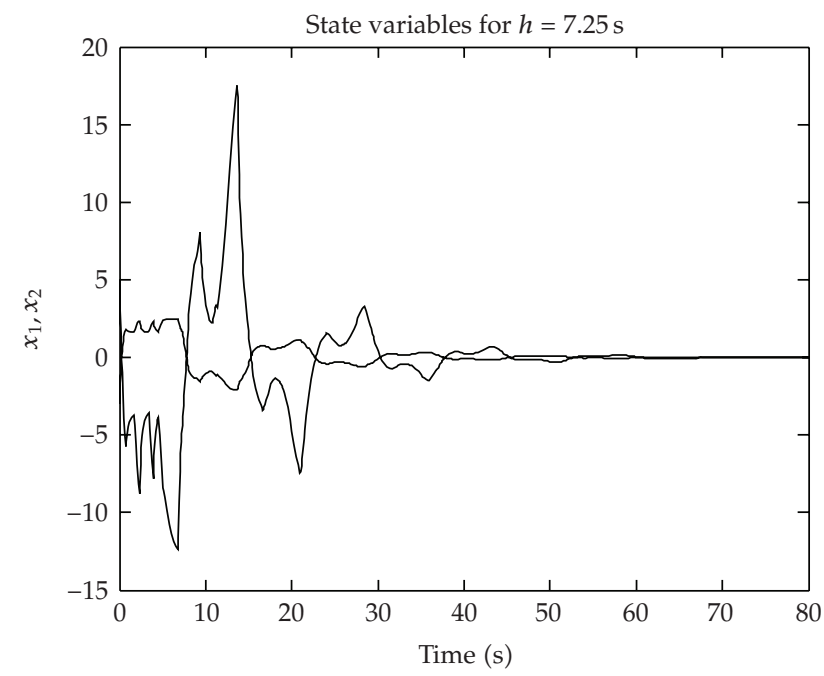

Figure 8: State trajectories evolution for a delay of 7.25 seconds.

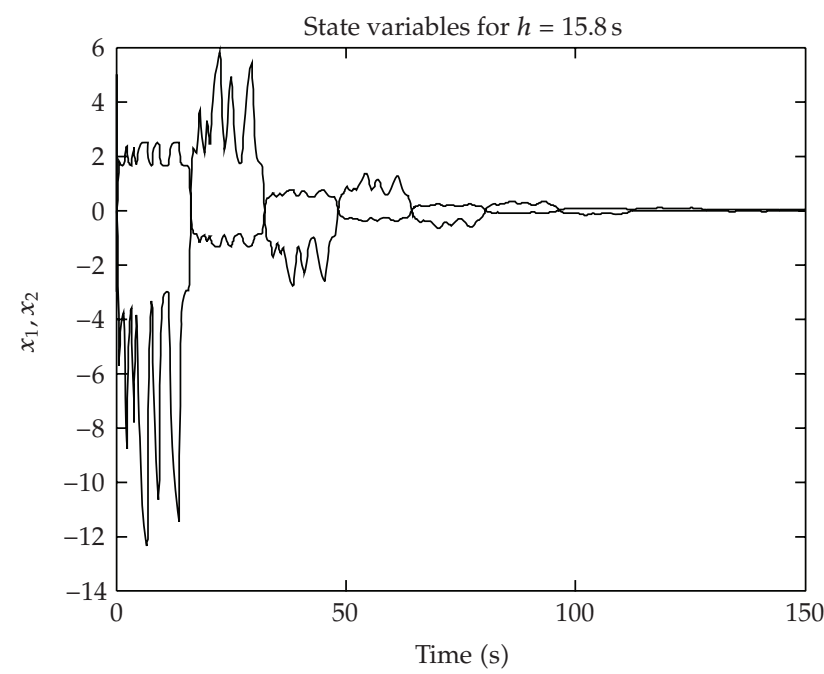

Figure 9: State trajectories evolution for a delay of 15.8 seconds.

\section{Conclusions}

This paper has been devoted to the investigation of the stability of switched linear timevarying systems with internal constant point delays. The switching laws are allowed to possess two kinds of switching instants, in general. The reset instants are those related to switching the current system parameterization to some configuration within a prescribed set. At switching time instants which are not reset instants, any bounded jump of any of the system parameter function associated either with the delay-free or with delayed dynamics for any of the delays is allowed. The system delay-free matrix as well as the matrices of delayed dynamics is allowed to be time-varying and eventually time differentiable. Also, either the delay-free system matrix or the system matrix obtained by zeroing the matrices of 


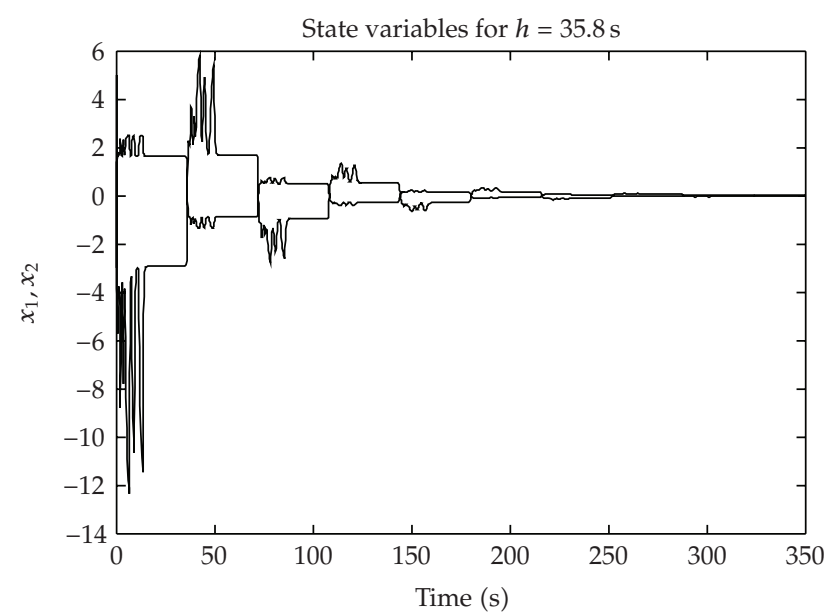

Figure 10: State trajectories evolution for a delay of 35.8 seconds.

dynamics of all nonzero delays are assumed to be stability matrices with prescribed stability abscissa for all time. The first assumption is used to obtain results for stability dependent on the sizes of the delays, while the second one is used for results concerning asymptotic stability independent of the delays. The parametrical bounded jumps at switching instants may be interpreted equivalently as Dirac impulses of the corresponding time derivatives. Global asymptotic stability and exponential stability results are obtained dependent on and independent of the sizes of the delays. Stability results are guaranteed based on the existence of a Krasovsky-Lyapunov functional through simple tests of negative definiteness of matrices for sufficiently small norms of the parametrical time derivatives, where such derivatives exist, compared to the above mentioned stability abscissas. In addition, the existence of a minimum residence time at each eventual resetting configuration is required to guarantee global asymptotic stability in the event that the Krasovsky-Lyapunov functional candidate has a positive jump at some reset switching instant.

\section{Appendix}

\section{A. Mathematical Proofs}

\section{A.1. Proof of Theorem 2.12}

(i) Denote by $x_{t}$ the strip of state-trajectory solution $x(t+\tau)$ of the system (2.1) for $\tau \in[-h, 0]$. Consider the Krasovsky-Lyapunov functional candidate:

$$
V\left(t, x_{t}\right)=x^{T}(t) P(t) x(t)+\sum_{i=1}^{q} \sum_{j=0}^{q} \int_{-h_{i}-h_{j}}^{-h_{j}} \int_{t+\theta}^{t} x^{T}(\tau) S_{i j} x(\tau) d \tau d \theta
$$

which is nonnegative and radially unbounded since $V\left(t, x_{t}\right) \rightarrow \infty$ as $\left\|x_{t}\right\|:=$ $\sup _{-h \leq \tau \leq 0}\|x(\tau)\| \rightarrow \infty$, with $h:=\max _{1 \leq i \leq q}\left(h_{i}\right)$, since all the eigenvalues of $P(t)$ and $S_{i j}(t)$ positive and uniformly bounded from above and below for all $t \in \mathbf{R}_{0+}$. This also implies that $\dot{P}(t)$ cannot be neither positive definite nor negative definite for all $t \in \mathbf{R}_{0+}$. Direct 
calculations via (2.9) yield $\dot{V}\left(t, x_{t}\right) \leq-\bar{x}^{T}(t) Q(t) \bar{x}(t)<0$, for all $t \in \mathbf{R}_{0+}$ if and only if $\bar{x}(t)=\left(x^{T}(t), x^{T}\left(t-h_{1}\right), \ldots, x^{T}\left(t-h_{q}\right)\right)^{T} \neq 0$. Then, $V\left(t, x_{t}\right) \leq V(0, \varphi)<\infty$ and $V\left(t, x_{t}\right) \rightarrow 0$ as $t \rightarrow \infty$ for any given bounded function of initial conditions what imply that $\left\|x_{t}\right\|<\infty$, for all $t \in \mathbf{R}_{0+}$ and $\left\|x_{t}\right\| \rightarrow 0$ as $t \rightarrow \infty$. The global asymptotic stability has been proven. To prove the last part of Property (i), note that (2.9) implies

$$
\begin{aligned}
& \left(\sum_{j=0}^{q} A_{j}^{T}(t)\right) P(t)+P(t)\left(\sum_{j=0}^{q} A_{j}(t)\right)+\dot{P}(t)+\sum_{i=1}^{q} \sum_{j=0}^{q} \widehat{h}_{i} S_{i j}<0, \quad \forall t \in \mathbf{R}_{0+} \\
& \Longrightarrow\left(\sum_{j=0}^{q} A_{j}^{T}(t)\right) P(t)+P(t)\left(\sum_{j=0}^{q} A_{j}(t)\right)+\dot{P}(t)<0 .
\end{aligned}
$$

Since $\dot{P}(t)<0$, for all $t \in \mathbf{R}_{0+}$ is impossible from the preceding part of the proof, it has to exist a nonnecessarily connected subinterval $S_{R} \subset \mathbf{R}_{0+}$ such that $\left(\sum_{j=0}^{q} A_{j}^{T}(t)\right) P(t)+$ $P(t)\left(\sum_{j=0}^{q} A_{i}(t)\right)<0$, for all $t \in S_{R}$. Now, proceed by contradiction to prove that $S_{R}$ has infinite measure with a connected component of infinite measure by assuming that the system (2.1) is globally asymptotically stable in the following cases.

(1) $S_{R}$ has finite measure so that the complement $\overline{S_{R}}$ in $\mathbf{R}_{0+}$ is nonconnected with infinite measure with a component being necessarily of infinite measure (otherwise, $S_{R}$ has infinite measure). Thus, $\left(\sum_{j=0}^{q} A_{j}^{T}(t)\right) P(t)+P(t)\left(\sum_{j=0}^{q} A_{j}(t)\right) \geq 0$ or indefinite for all $t \in S_{R}$. Since $S_{R}$ has finite measure and $\overline{S_{R}}$ has a component of infinite measure, it exists a sufficiently large finite $t_{0} \in \mathbf{R}_{0+}$ such that $\overline{S_{R}} \ni t \geq t_{0}$ so that $\left\|x_{t}\right\| \rightarrow 0$ as $t \rightarrow \infty$ is impossible what leads to a contradiction.

(2) Both intervals $S_{R}$ and $\overline{S_{R}}$ have infinite measures so that they are nonconnected and have infinite components each of them with finite measure. Thus, asymptotic stability is also impossible.

As conclusion, $\left(\sum_{j=0}^{q} A_{j}(t)\right)$ is a stability matrix for all $t \in \mathbf{R}_{0+}$ except possibly within an interval of finite measure.

(ii) Denote by $z_{t}$ the strip of state-trajectory solution $z(t+\tau)$, for $\tau \in[-h, 0]$ and any resetting system. Consider the Krasovsky-Lyapunov functional candidate for all the resetting systems:

$$
V^{*}\left(t, z_{t}\right)=z^{T}(t) P^{*} z(t)+\sum_{i=1}^{q} \sum_{j=0}^{q} \int_{-h_{i}-h_{j}}^{-h_{j}} \int_{t+\theta}^{t} z^{T}(\tau) S_{i j}^{*} z(\tau) d \tau d \theta
$$

The real functional (A.1) is a common Krasovsky-Lyapunov functional for all $p$ distinct resetting systems for all delays $h_{i} \in\left[0, \widehat{h}_{i}\right](\forall i \in \bar{q})$, provided that $z_{t} \equiv z(t+\tau)$, for all $\tau \in$ $[-\widehat{h}, 0]$ and $\widehat{h}:=\max _{1 \leq i \leq q}\left(\widehat{h}_{i}\right)$ since the $i$ th resetting system (2.4) satisfies from (2.11)

$$
\dot{V}_{i}^{*}\left(t, z_{t}\right) \leq \bar{z}^{T}(t) Q_{i}^{*} \bar{z}(t)<0 ; \quad \forall i \in \bar{q}, \forall t \in \mathbf{R}_{0+}
$$


for all nonzero $\bar{z}(t)$ where $\bar{z}(t):=\left(z^{T}(t), z^{T}\left(t-h_{1}\right), \ldots, z^{T}\left(t-h_{q}\right)\right)^{T}$ and accordingly

$$
\dot{V}^{*}\left(t, x_{t}\right) \leq-\left|\min _{i \in \bar{q}} \bar{x}^{T}(t) Q_{i}^{*} \bar{x}(t)\right| \leq 0, \quad \forall t \in \mathbf{R}_{0+}
$$

for all nonzero $\bar{x}(t)$ where $\bar{x}(t):=\left(x^{T}(t), x^{T}\left(t-h_{1}\right), \ldots, x^{T}\left(t-h_{q}\right)\right)^{T}$ for the switched system (2.1) since

$$
\begin{aligned}
\operatorname{ST}_{\mathrm{rf}}(\sigma)=\varnothing & \Longrightarrow \mathrm{ST}_{r}(\sigma)=\mathrm{ST}_{r}(\sigma) \\
& \Longrightarrow-\left(\max _{i \in \bar{p}} \lambda_{\max }\left(Q_{i}^{*}\right)\right) \bar{z}^{T}(t) \bar{z}(t) \leq \dot{V}^{*}\left(t, z_{t}\right) \\
& \leq-\left(\min _{i \in \bar{p}} \lambda_{\min }\left(Q_{i i}^{*}\right)\right) \bar{z}^{T}(t) \bar{z}(t)<0
\end{aligned}
$$

for any $t \in \mathbf{R}_{0+}$ such that $z(t) \neq 0$, where $\lambda_{\max }(\cdot)$ and $\lambda_{\min }(\cdot)$ stand for maximum and minimum eigenvalues of real symmetric matrices. Thus, if (2.9) holds then the candidate (A.3) is a common Krasovsky-Lyapunov functional for all the resetting systems, and then for the switched system (2.1) which is then globally asymptotically Lyapunov's stable. Furthermore, the Krasovsky-Lyapunov functional of the switched system (2.1) fulfils from (A.3)-(A.6):

$$
\frac{\dot{V}^{*}\left(t, x_{t}\right)}{V^{*}\left(t, x_{t}\right)} \leq-\delta<0 \Longrightarrow V^{*}\left(t, x_{t}\right) \leq e^{-\delta T} V^{*}\left(t-T, x_{t-T}\right) \leq K e^{-\delta t} V^{*}(0, \varphi)
$$

for all $t \in \mathbf{R}_{0+}$, for some finite $K \in \mathbf{R}_{+}$, where $\delta:=\left|\max _{i \in \bar{p}} \lambda_{\max }\left(Q_{i}^{*}\right)\right| / \lambda_{\min }\left(P^{*}\right)>0$. Then, from (A.7) and (A.3)

$$
\begin{aligned}
& \lambda_{\min }\left(P^{*}\right) x^{T}(t) x(t) \leq V^{*}\left(t, x_{t}\right) \leq e^{-\delta T} V^{*}\left(t-T, x_{t-T}\right) \leq K e^{-\delta t} V^{*}(0, \varphi) \\
& \leq K K_{1} e^{-\delta t} \sup _{-\hat{h} \leq \tau \leq 0}\|\varphi(\tau)\|_{2}^{2} \\
& \Longrightarrow\|x(t)\|_{2} \leq \sqrt{K K_{1}} e^{-(\delta / 2) t} \sup _{-\hat{h} \leq \tau \leq 0}\|\varphi(\tau)\|_{2}
\end{aligned}
$$

for all $t \in \mathbf{R}_{0+}$, and some $K \in \mathbf{R}_{+}$, where $K_{1}:=\lambda_{\max }\left(P^{*}\right)+(q+1) \max _{i \in \bar{p}, j \in \bar{q} \cup\{0\}} \lambda_{\max }\left(S_{i j}\right)$ and $\widehat{h}:=$ $\max _{i \in \bar{q}} \widehat{h}_{i}$ and $\|\cdot\|_{2}$ denotes the $\ell_{2}$ (or spectral) vector norm or the corresponding induced ones for matrices. Then, the system (2.1) is globally exponentially stable for all delays $h_{i} \in\left[0, \widehat{h}_{i}\right]$, for all $i \in \bar{q}$. The modification of (2.11)-(2.12) with $Q_{i} \leq-2 \varepsilon I_{(q+1) n}<0$, for all $i \in \bar{q}$ leads directly to an exponential decay of $\|x(t)\|_{2}$ with rate $\delta=2 \varepsilon$ from a similar slightly extended proof.

(iii) If Assumption 2.4 holds then $\left(\sum_{j=0}^{q} A_{j i}^{T}\right) P^{*}+P^{*}\left(\sum_{j=0}^{q} A_{j i}\right)<0$ for any $P^{*}=P^{* T}>0$. Thus, it exists a sufficiently small $\widehat{h}:=\max _{i \in \bar{q}} \widehat{h}_{i}$ such that (2.9) holds for all $h_{i} \in\left[0, \widehat{h}_{i}\right]$, since $R_{i}^{*}>0$; for all $i \in \bar{q}$.

If Property (i) holds then $\left(\sum_{j=0}^{q} A_{j i}^{T}\right) P^{*}+P^{*}\left(\sum_{j=0}^{q} A_{j i}\right)+\sum_{i=1}^{q} \sum_{j=0}^{q} \widehat{h}_{i} S_{i j}<0$ what implies $\left(\sum_{j=0}^{q} A_{j i}^{T}\right) P^{*}+P^{*}\left(\sum_{j=0}^{q} A_{j i}\right)<0$ since $\sum_{i=1}^{q} \sum_{j=0}^{q} \widehat{h}_{i} S_{i j} \geq 0$ for any $\widehat{h}_{i} \geq 0$, for all $i \in \bar{q}$. Thus, 
$Q_{\text {id }}:=$ Block Diag $\left(\left(\sum_{j=0}^{q} A_{j i}^{T}\right) P^{*}+P^{*}\left(\sum_{j=0}^{q} A_{j i}\right)+\sum_{i=1}^{q} \sum_{j=0}^{q} \widehat{h}_{i} S_{i j}<0,-R_{1}^{*}, \ldots, \quad-R_{q}^{*}\right)<0$, for all $i \in \bar{q}$ and then $Q_{i}<0$, for all $h_{i} \in\left[0, \widehat{h}_{i}\right]$ and sufficiently small $\widehat{h}:=\max _{i \in \bar{q}} \widehat{h}_{i}$ since from (2.11) $\left\|Q_{i}-Q_{\text {id }}\right\|$ is a monotonically increasing function of the argument $\left(\widehat{h}_{1}, \ldots, \widehat{h}_{q}\right)$ being zero if $\widehat{h}_{i}=0$, for all $i \in \bar{q}$. Then, Property (i) holds for a sufficiently small $\widehat{h}$.

\section{A.2. Derivation of the inequality (2.17)}

It follows from the subsequent inequalities:

$$
\begin{aligned}
&\left\|M^{*}\right\|_{2} \leq \sum_{i=0}^{q}\left\|A_{i}^{*}\right\|_{2^{\prime}} \quad\|M(t)\|_{2} \leq \sum_{i=0}^{q}\left\|A_{i}(t)\right\|_{2^{\prime}} \\
&\left\|M\left(t^{+}\right)-M(t)\right\|_{2} \leq \sum_{i=0}^{q}\left\|A_{i}\left(t^{+}\right)-A_{i}(t)\right\|_{2^{\prime}} \quad\left\|M\left(t^{+}\right)-M^{*}\right\|_{2} \leq \sum_{i=0}^{q}\left\|A_{i}\left(t^{+}\right)-A_{i}^{*}\right\|_{2^{\prime}} \\
&\left\|\widetilde{\Delta}_{i}\left(t^{+}\right)-\tilde{\Delta}_{i}(t)\right\|_{2}= \|\left(\tilde{A}_{i}\left(t^{+}\right)-\widetilde{A}_{i}(t)\right) M^{*}+A_{i}^{*}\left(M\left(t^{+}\right)-M(t)\right) \\
&+\left(\tilde{A}_{i}\left(t^{+}\right)\left(M\left(t^{+}\right)-M^{*}\right)+\tilde{A}_{i}(t)\left(M(t)-M^{*}\right)\right) \|_{2} \\
& \leq\left\|A_{i}\left(t^{+}\right)-A_{i}(t)\right\|_{2}\left(\sum_{j=0}^{q}\left\|A_{j}^{*}\right\|_{2}\right)+\left\|A_{i}^{*}\right\|_{2}\left\|\left(\sum_{j=0}^{q}\left\|A_{j}\left(t^{+}\right)-A_{j}(t)\right\|_{2}\right)\right\|_{2} \\
&+\left\|A_{i}\left(t^{+}\right)-A_{i}^{*}\right\|_{2}\left(\sum_{j=0}^{q}\left\|A_{j}\left(t^{+}\right)-A_{j}^{*}\right\|_{2}\right) \\
&+\left\|A_{i}(t)-A_{i}^{*}\right\|_{2}\left(\sum_{j=0}^{q}\left\|A_{j}(t)-A_{j}^{*}\right\|_{2}\right) \\
& \leq a^{+}\left(\sum_{j=0}^{q}\left\|A_{j}^{*}\right\|_{2}+(q+1)\left\|A_{i}^{*}\right\|_{2}+(q+1) a^{+}+\sum_{j=0}^{q}\left\|A_{j}(t)-A_{j}^{*}\right\|_{2}\right) \\
&+\left\|A_{i}(t)-A_{i}^{*}\right\|_{2}\left[(q+1) a^{+}+\sum_{j=0}^{q}\left\|A_{j}(t)-A_{j}^{*}\right\|_{2}+\sum_{j=0}^{q}\left\|A_{j}(t)-A_{j}^{*}\right\|_{2}\right] \\
& \leq a^{+}\left(\sum_{j=0}^{q}\left\|A_{j}^{*}\right\|_{2}+(q+1)\left\|A_{i}^{*}\right\|_{2}+(q+1)\left(a+a^{+}\right)\right) \\
&+(q+1)\left(2 a+a^{+}\right) a, \forall i \in \bar{q} \cup\{0\} .
\end{aligned}
$$

Proof of Theorem 2.16. (i) It can be considered by the obvious nature of the process that the switching set ST $(\sigma)$ of the switching law $\sigma$ is defined by the discrete set of times where the time derivative of some of the entries of some of the delay-free or delayed matrices of dynamics does not exist, or equivalently, is impulsive which translated in a bounded discontinuity of the corresponding matrix function at such a time instant. Conversely, a bounded discontinuity of any of such matrices is equivalent to a distributional time derivative. Thus, if Assumptions 2.1-2.3 hold then $\left(\sum_{j=0}^{q} A_{j}(t)\right)$ is a stability matrix, 
for all $t \in \mathbf{R}_{0+}$, so that a positive definite symmetric $P(t)$ has to exist as a unique solution to the following matrix Lyapunov equation $Q(t)$ in (2.9) takes the form

$$
\left(\sum_{j=0}^{q} A_{j}^{T}(t)\right) P(t)+P(t)\left(\sum_{j=0}^{q} A_{j}(t)\right)=-I_{n}
$$

where $I_{n}$ is the $n$th identity matrix. Taking time-derivatives in (A.9) yields to the subsequent matrix Lyapunov equation:

$$
\left(\sum_{j=0}^{q} A_{j}^{T}(t)\right) \dot{P}(t)+\dot{P}(t)\left(\sum_{j=0}^{q} A_{j}(t)\right)=-\left(\sum_{j=0}^{q} \dot{A}_{j}^{T}(t)\right) P(t)-P(t)\left(\sum_{j=0}^{q} \dot{A}_{j}(t)\right) .
$$

Thus, the unique solutions to the above Lyapunov equations are, respectively,

$$
\begin{gathered}
P(t)=\int_{0}^{\infty} e^{\left(\sum_{j=0}^{q} A_{j}^{T}(t)\right) \tau} e^{\left(\sum_{j=0}^{q} A_{j}(t)\right) \tau} d \tau \\
\dot{P}(t)=\int_{0}^{\infty} e^{\left(\sum_{j=0}^{q} A_{j}^{T}(t)\right) \tau}\left(\left(\sum_{j=0}^{q} \dot{A}_{j}^{T}(t)\right) P(t)+P(t)\left(\sum_{j=0}^{q} \dot{A}_{j}(t)\right)\right) e^{\left(\sum_{j=0}^{q} A_{j}(t)\right) \tau} d \tau
\end{gathered}
$$

with

$$
\begin{gathered}
\lambda_{\max }(P(t))=\|P(t)\|_{2} \leq \frac{K_{0}}{2 \rho_{0}} \\
\left|\lambda_{\max }(\dot{P}(t))\right|=\|\dot{P}(t)\|_{2} \leq \frac{K_{0} \lambda_{\max }(P(t))}{\rho_{0}}\left\|\sum_{j=0}^{q} \dot{A}_{j}(t)\right\|_{2} \leq \frac{K_{0}^{2}}{2 \rho_{0}^{2}}\left\|\sum_{j=0}^{q} \dot{A}_{j}(t)\right\|_{2} \leq \frac{K_{0}^{2} \gamma_{2}}{2 \rho_{0}^{2}},
\end{gathered}
$$

provided that $\sum_{j=0}^{q}\left\|\dot{A}_{j}(t)\right\|_{2} \leq \gamma_{2} \in \mathbf{R}_{0+}$, from Assumptions 2.3 .

Remark A.1. Note that $P(t),($ A.12), may be (pointwise) equivalently calculated from the linear algebraic system below of $n^{2}$ unknowns (the entries of $P(t)$ at each time) and $n^{2} \times n^{2}$ coefficient matrix which is equivalent to the matrix equation (A.10):

$$
\begin{aligned}
& {\left[\left(\sum_{j=0}^{q} A_{j}^{T}(t)\right) \otimes I_{n}+I_{n} \otimes\left(\sum_{j=0}^{q} A_{j}^{T}(t)\right)\right] \operatorname{vec}(P(t))} \\
& \quad=\operatorname{vec}\left(-I_{n}\right)=-\left(e_{1}^{T}, e_{1}^{T}, \ldots, e_{1}^{T}\right)^{T} \\
& \Longleftrightarrow \operatorname{vec}(P(t))=\left[\left(\sum_{j=0}^{q} A_{j}^{T}(t)\right) \otimes I_{n}+I_{n} \otimes\left(\sum_{j=0}^{q} A_{j}^{T}(t)\right)\right]^{-1} \operatorname{vec}\left(-I_{n}\right)^{T},
\end{aligned}
$$


where " $\otimes$ " defines the Kronecker (or direct) product of matrices and $\operatorname{vec}(M):=$ $\left(m_{1}^{T}, m_{2}^{T}, \ldots, m_{n}^{T}\right)^{T} \in \mathbf{R}^{n m}$ if $M=\left(m_{1}, m_{2}, \ldots, m_{n}\right)^{T}$ is an $n \times m$ real matrix of rows $m_{i}^{T}(i \in \bar{n})$ each of $m$ components. Note that the coefficient matrix of the above algebraic system is everywhere nonsingular since $P(t)$ exists and it is unique for all time.

Thus, one gets from (2.9) that $Q(t) \leq \widehat{Q}(t)$, for all $t \in \mathbf{R}_{0+} \backslash \mathrm{ST}(\sigma)$, where

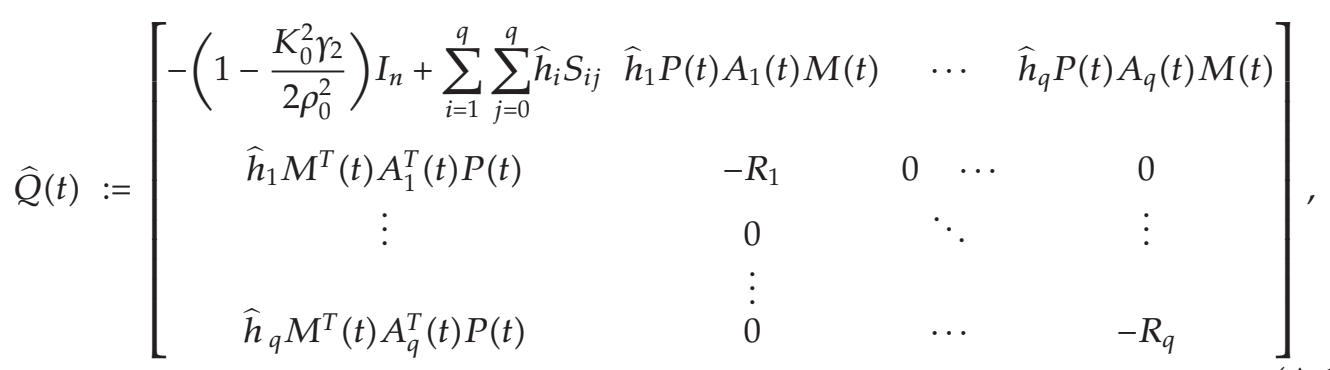

provided that $\widehat{h}$ is sufficiently small and $2\left(\rho_{0} / K_{0}\right)^{2}>\gamma_{2} \geq \operatorname{ess} \sup _{t \in \mathbf{R}_{0+} \backslash \mathrm{ST}(\sigma)}\left\|\sum_{j=0}^{q} \dot{A}_{j}(t)\right\|_{2}$. On the other hand, one has for $t \in \mathrm{ST}(\sigma)$

$$
\widehat{Q}\left(t^{+}\right):=\left[\begin{array}{cccc}
\tilde{P}(t) \delta(0) & \widehat{h}_{1} P(t) A_{1}\left(t^{+}\right) M\left(t^{+}\right) & \ldots & \widehat{h}_{q} P(t) A_{q}\left(t^{+}\right) M\left(t^{+}\right) \\
\widehat{h}_{1} M^{T}\left(t^{+}\right) A_{1}^{T}\left(t^{+}\right) P(t) & 0 & 0 & \ldots \\
\vdots & 0 & \ddots & 0 \\
\widehat{h}_{q} M^{T}\left(t^{+}\right) A_{q}^{T}\left(t^{+}\right) P(t) & \vdots & & \vdots
\end{array}\right],
$$

where $\delta(0)$ is the unity scalar Dirac distribution centered at $t=0$ and $\widetilde{P}(t)=\dot{P}\left(t^{+}\right)-\dot{P}(t)$ subject to

$$
\begin{aligned}
P\left(t^{+}\right)=\int_{0}^{\infty} e^{\left(\sum_{j=0}^{q} A_{j}^{T}\left(t^{+}\right)\right) \tau} e^{\left(\sum_{j=0}^{q} A_{j}\left(t^{+}\right)\right) \tau} d \tau \\
\dot{P}\left(t^{+}\right)=\int_{0}^{\infty} e^{\left(\sum_{j=0}^{q} A_{j}^{T}\left(t^{+}\right)\right) \tau}\left(\left(\sum_{j=0}^{q}\left(\dot{A}_{j}^{T}(t)+\Gamma_{A d j}^{T}(t)\right)\right) P(t)\right. \\
\left.+P(t)\left(\sum_{j=0}^{q}\left(\dot{A}_{j}(t)+\Gamma_{A d j}(t)\right)\right)\right) e^{\left(\sum_{j=0}^{q} A_{j}\left(t^{+}\right)\right) \tau} d \tau
\end{aligned}
$$


so that

$$
\begin{array}{cl}
\left|\lambda_{\max }(\dot{P}(t))\right| \leq \frac{K_{0}^{2} \gamma_{2}}{2 \rho_{0}^{2}} ; & \left|\lambda_{\max }\left(\dot{P}\left(t^{+}\right)\right)\right| \leq \frac{K_{0}^{2}\left(\gamma_{2}+\sum_{j=0}^{q}\left\|\Gamma_{A d_{j}}(t)\right\|_{2} \delta(0)\right)}{2 \rho_{0}^{2}}, \\
0<\lambda_{\max }(P(t)) \leq \frac{K_{0}}{2 \rho_{0}} ; & 0<\lambda_{\max }\left(P\left(t^{+}\right)\right) \leq \frac{K_{0}\left(1+\sum_{j=0}^{q}\left\|\Gamma_{A d_{j}}(t)\right\|_{2}\right)}{2 \rho_{0}},
\end{array}
$$

with $\Gamma_{A d_{j}}(t):=A_{j}\left(t^{+}\right)-A_{j}(t)$, or equivalently $\dot{A}_{j}\left(t^{+}\right)-\dot{A}_{j}(t)=\Gamma_{A d_{j}}(t) \delta(0)$, being a real $n$-matrix whose entries are zero if $A_{j}(t)$ is continuous at time $t$ and each nonzero entry is the amplitude of any impulse at the time derivative. Thus, one gets from (A.18)

$$
\begin{aligned}
\widehat{Q}\left(t^{+}\right) & -\widehat{Q}(t) \\
& \leq\left[\begin{array}{ccccc}
\frac{K_{0}^{2}\left(\sum_{j=0}^{q}\left\|\Gamma_{A d_{j}}(t)\right\|_{2}\right)}{2 \rho_{0}^{2}} \delta(0) I_{n} & \widehat{h}_{1} \vartheta_{1} \lambda_{\max }(P(t)) E & \ldots & \widehat{h}_{q} \vartheta_{q} \lambda_{\max }(P(t)) E \\
\widehat{h}_{1} \vartheta_{1} \lambda_{\max }(P(t)) E & 0 & 0 & \ldots & 0 \\
\vdots & 0 & \ddots & \vdots \\
\widehat{h}_{q} \vartheta_{q} \lambda_{\max }(P(t)) E & \vdots & &
\end{array}\right],
\end{aligned}
$$

where $\vartheta_{i} \leq\left\|\Gamma_{A d_{i}}(t)\right\|_{2}\left(\sum_{j=0}^{q}\left\|\Gamma_{A d_{j}}(t)\right\|_{2}\right)$, for all $i \in \bar{q} \cup\{0\}$ and $E$ is a real $n \times(q+1) n$-matrix with all its entries being unity. Since $\widehat{Q}(t)<0$, it follows from (A.21) that if the matrix $\sum_{j=0}^{q} \Gamma_{A d_{j}}(t) \leq 0$, for all $t \in \mathrm{ST}(\sigma)$, then the $(1,1)$ block-matrix in (A.18) is semidefinite negative for any (sufficiently small) $\hat{h}$ compared to $\left(\sum_{j=0}^{q}\left\|\Gamma_{A d_{j}}(t)\right\|_{2}\right)$ and $\vartheta:=\max \left(\vartheta_{i}: i \in\right.$ $\bar{q} \cup\{0\})$ provided that $2\left(\rho_{0} / K_{0}\right)^{2}>\gamma_{2} \geq \operatorname{ess} \sup _{t \in \mathbf{R}_{0+} \backslash \operatorname{ST}(\sigma)}\left\|\sum_{j=0}^{q} \dot{A}_{j}(t)\right\|_{2^{\prime}}$ for all $t \in \mathbf{R}_{0+} \backslash \mathrm{ST}(\sigma)$. Thus, Property (i) has been proven.

(ii) From (2.9) and (A.15),

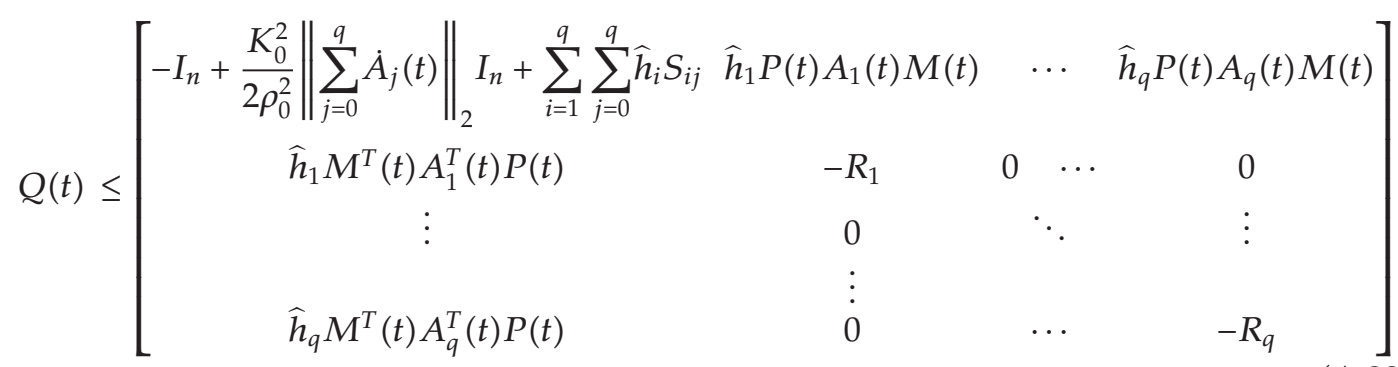


so that if $Q^{\prime}(t):=Q(t)-\operatorname{Block} \operatorname{Diag}\left(\left(K_{0}^{2} / 2 \rho_{0}^{2}\left\|\sum_{j=0}^{q} \dot{A}_{j}(t)\right\|-1\right) I_{n}, 0, \ldots, 0\right)$,

$$
\begin{aligned}
\dot{V}\left(t, x_{t}\right) \leq & \left(\frac{K_{0}^{2}}{2 \rho_{0}^{2}}\left\|\sum_{j=0}^{q} \dot{A}_{j}(t)\right\|_{2}+\sum_{i=1}^{q} \sum_{j=0}^{q} \widehat{h}_{i}\left\|S_{i j}(t)\right\|_{2}-1\right)\|x(t)\|_{2}^{2}-\sum_{i=1}^{q}\left\|x\left(t-h_{i}\right)\right\|_{R_{i}(t)}^{2} \\
& +\left(\frac{K_{0}}{\rho_{0}}\right)^{2} \sum_{i=1}^{q} \sum_{j=0}^{q} \widehat{h}_{i}\left\|A_{i}(t)\right\|_{2}\left\|A_{j}(t)\right\|_{2}\left\|x\left(t-h_{i}\right)\right\|_{2}\|x(t)\|_{2} \\
\leq & \left(\frac{K_{0}^{2} \gamma_{2}}{2 \rho_{0}^{2}}-1\right)\|x(t)\|_{2}^{2}-\sum_{i=1}^{q}\left\|x\left(t-h_{i}\right)\right\|_{R_{i}(t)}^{2} \\
& +\widehat{h}\left[\left(\frac{K_{0}}{\rho_{0}}\right)^{2} \sum_{i=1}^{q} \sum_{j=0}^{q}\left\|A_{i}(t)\right\|_{2}\left\|A_{j}(t)\right\|_{2}\left\|x\left(t-h_{i}\right)\right\|_{2}+\sum_{i=1}^{q} \sum_{j=0}^{q}\left\|S_{i j}(t)\right\|_{2}\|x(t)\|_{2}\right]\|x(t)\|_{2} \\
\leq & \left(\frac{K_{0}^{2} \gamma_{2}}{2 \rho_{0}^{2}}-1\right) \frac{V\left(t, x_{t}\right)}{\lambda_{\min }(P(t))}+o(\widehat{h}) \max _{0 \leq i \leq q}\left\|x\left(t-h_{i}\right)\right\|_{2}^{2} \\
\leq & \left(\frac{K_{0}^{2} \gamma_{2}}{2 \rho_{0}^{2}}-1+o(\widehat{h})\right) \frac{\max _{0 \leq i \leq q} V\left(t-h_{i}, x_{t-h_{i}}\right)}{\min _{0 \leq i \leq q}\left(\lambda_{\min }\left(P\left(t-h_{i}\right)\right)\right)} \\
\leq & \frac{1}{\beta}\left(\frac{K_{0}^{2} \gamma_{2}}{2 \rho_{0}^{2}}-1+o(\widehat{h})\right) V\left(t, x_{t}\right),
\end{aligned}
$$

for all $t \in \mathbf{R}_{0}^{+} \backslash \mathrm{ST}(\sigma)$, since $0 \leq \lambda_{\min }(P(t))\|x(t)\|_{2}^{2} \leq V\left(t, x_{t}\right) \leq V\left(t^{\prime}, x_{t^{\prime}}\right) \leq V(0, \varphi)<\infty$ with $\dot{V}\left(t, x_{t}\right) \leq 0$, for all $t\left(\geq t^{\prime}\right), t^{\prime} \in \mathbf{R}_{0+}$ from the properties of the Krasovsky-Lyapunov functional Equation (A.1) used in the proof of Theorem 2.12, where $\mathbf{R}_{+} \ni \beta:=\inf _{t \in \mathbf{R}_{0_{+}}} \lambda_{\min }(P(t))$ since $P(t)>0$, for all $t \in \mathbf{R}_{0+}$ and the "small-o" and "big-O" Landau's notations mean the following:

$$
f=o(s) \Longleftrightarrow f=O(s)\left(\Longleftrightarrow|f| \leq k_{1}|s|+k_{2} \text {, some } k_{1,2} \in \mathbf{R}_{0+}\right) \wedge \exists \lim _{s \rightarrow 0}\left|\frac{f}{s}\right|=0 .
$$

Thus, if $\gamma_{2} \leq 2\left(\rho_{0} / K_{0}\right)(1-\varepsilon)$ for some $\varepsilon \in \mathbf{R}_{+}$and $\widehat{h}$ is sufficiently small then one gets from (A.23) that

$$
\begin{aligned}
\left\|x\left(t^{+}\right)\right\|_{2}^{2} & \leq \frac{V\left(t^{+}, x_{t^{+}}\right)}{\beta} \leq K_{V} e^{-\zeta T_{k}} V\left(t^{+}-T_{k}, x_{t^{+}-T_{k}}\right) \\
& \leq K_{V} e^{-\zeta T_{k}}\left(V\left(t-T_{k}, x_{t-T_{k}}\right)+\left(V\left(t^{+}-T_{k}, x_{t^{+}-T_{k}}\right)-V\left(t-T_{k}, x_{t-T_{k}}\right)\right)\right) \\
& \leq K_{V} \bar{K} e^{-\zeta t_{k}}\left(\prod_{i=1}^{k} \eta\left(t_{i}\right)\right) \max _{0 \leq i \leq q}\left\|x\left(t-t_{k}-h_{i}\right)\right\|_{2}^{2}<\infty \\
& \leq K_{V} \bar{K} e^{-\zeta t_{k}}(1+\bar{\eta})^{k} \max _{0 \leq i \leq q}\left\|x\left(t-t_{k}-h_{i}\right)\right\|_{2}^{2}<\infty .
\end{aligned}
$$


With the following.

(a) $K_{V}, K \in \mathbf{R}_{+}$being some constants independent of $t$.

(b) ST $(\sigma)=\left\{t_{i}: i \in \mathbf{N}\right\}$ is defined such that it is a strictly ordered set of switching instants, $t \in\left(t_{k}, t_{k+1}\right] \cap \mathbf{R}_{+}$provided that $t_{k}<t_{k+1} \in \mathrm{ST}(\sigma)$ exists so that, $T_{k}:=t_{k}-t_{k-1}$ (i.e., $\left.t_{k}=\sum_{i=1}^{k} T_{i}\right)$, and $t \in\left(t_{k}, \infty\right)$ for any $T_{k} \in \mathbf{R}_{+}$and some $k \in \mathbf{N}$, otherwise, (i.e., if $t_{k}$ is the existing maximal element in the set $\mathrm{ST}(\sigma)$ provided that it is of finite cardinal)

$$
\eta\left(t_{i}\right) \leq 1+\bar{\eta} \quad \text { with } \bar{\eta}:=\max \left(V\left(t^{+}-T_{k}, x_{t^{+}-T_{k}}\right)-V\left(t-T_{k}, x_{t-T_{k}}\right): t \in \mathbf{R}_{0+}\right) .
$$

Thus, $\lim _{t \rightarrow \infty}\|x(t)\|_{2}^{2}=0$ with exponential convergence rate for any admissible function of initial conditions of the state-trajectory solution $\varphi:[-h, 0] \rightarrow \mathbf{R}^{n}$ provided that for some $\varepsilon_{0}(<1) \in \mathbf{R}_{+}:-\zeta t_{k}(1+\bar{\eta})^{k} \leq 1-\varepsilon_{0} \Leftrightarrow 1 \leq \ln (1+\bar{\eta}) \leq\left(\zeta t_{k}+\ln \left(1-\varepsilon_{0}\right)\right) / k$ which is guaranteed, in particular, if $T_{k}=t_{k}-t_{k-1} \geq(1 / \zeta) \ln \left((1+\bar{\eta}) /\left(1-\varepsilon_{0}\right)\right)$. Then, Property (ii) follows directly.

(iii) The proof is direct since either Condition (b.1) or Condition (b.2) allow to derive a similar proof as that proof Property (ii).

\section{Acknowledgments}

The authors are very grateful to the Spanish Ministry of Education for its partial support of this work through Project DPI2006-00714. They are also grateful to the Basque Government for its support through GIC07143-IT-269-07, SAIOTEK SPED06UN10, and SPE07UN04.

\section{References}

[1] S.-I. Niculescu, Delay Effects on Stability: A Robust Control Approach, vol. 269 of Lecture Notes in Control and Information Sciences, Springer, London, UK, 2001.

[2] M. de la Sen, "Stability criteria for linear time-invariant systems with point delays based on onedimensional Routh-Hurwitz tests," Applied Mathematics and Computation, vol. 187, no. 2, pp. 11991207, 2007.

[3] M. da la Sen, "Quadratic stability and stabilization of switched dynamic systems with uncommensurate internal point delays," Applied Mathematics and Computation, vol. 185, no. 1, pp. 508-526, 2007.

[4] M. de la Sen, "Robust stability analysis and dynamic gain-scheduled controller design for point timedelay systems with parametrical uncertainties," Communications in Nonlinear Science and Numerical Simulation, vol. 13, no. 6, pp. 1131-1156, 2008.

[5] A. Ibeas and M. de la Sen, "Robustly stable adaptive control of a tandem of master-slave robotic manipulators with force reflection by using a multiestimation scheme," IEEE Transactions on Systems, Man and Cybernetics, Part B, vol. 36, no. 5, pp. 1162-1179, 2006.

[6] H. K. Lam and F. H. F. Leung, "Stability analysis of discrete-time fuzzy-model-based control systems with time delay: time delay-independent approach," Fuzzy Sets and Systems, vol. 159, no. 8, pp. 9901000, 2008.

[7] W. Qi and B. Dai, "Almost periodic solution for $n$-species Lotka-Volterra competitive system with delay and feedback controls," Applied Mathematics and Computation, vol. 200, no. 1, pp. 133-146, 2008.

[8] A. Maiti, A. K. Pal, and G. P. Samanta, "Effect of time-delay on a food chain model," Applied Mathematics and Computation, vol. 200, no. 1, pp. 189-203, 2008.

[9] A. U. Afuwape and M. O. Omeike, "On the stability and boundedness of solutions of a kind of third order delay differential equations," Applied Mathematics and Computation, vol. 200, no. 1, pp. 444-451, 2008.

[10] M. de la Sen and N. Luo, "On the uniform exponential stability of a wide class of linear time-delay systems," Journal of Mathematical Analysis and Applications, vol. 289, no. 2, pp. 456-476, 2004.

[11] X. Lou and B. Cui, "Existence and global attractivity of almost periodic solutions for neural field with time delay," Applied Mathematics and Computation, vol. 200, no. 1, pp. 465-472, 2008. 
[12] J. H. Park and O. M. Kwon, "On improved delay-dependent criterion for global stability of bidirectional associative memory neural networks with time-varying delays," Applied Mathematics and Computation, vol. 199, no. 2, pp. 435-446, 2008.

[13] Z. Wang, H. Zhang, and W. Yu, "Robust exponential stability analysis of neural networks with multiple time delays," Neurocomputing, vol. 70, no. 13-15, pp. 2534-2543, 2007.

[14] Z. H. Wang, "Numerical stability test of neutral delay differential equation," Mathematical Problems in Engineering, vol. 2008, Article ID 698043, 10 pages, 2008.

[15] D. Chen and W. Zhang, "Sliding mode control of uncertain neutral stochastic systems with multiple delays," Mathematical Problems in Engineering, vol. 2008, Article ID 761342, 9 pages, 2008.

[16] C. Wei and L. Chen, "A delayed epidemic model with pulse vaccination," Discrete Dynamics in Nature and Society, vol. 2008, Article ID 746951, 12 pages, 2008.

[17] Q. Liu, “Almost periodic solution of a diffusive mixed system with time delay and type III functional response," Discrete Dynamics in Nature and Society, vol. 2008, Article ID 706154, 13 pages, 2008.

[18] M. de la Sen, "On minimal realizations and minimal partial realizations of linear time-invariant systems subject to point incommensurate delays," Mathematical Problems in Engineering, vol. 2008, Article ID 790530, 19 pages, 2008.

[19] Z. Li, Y. Soh, and C. Wen, Switched and Impulsive Systems: Analysis, Design, and Applications, vol. 313 of Lecture Notes in Control and Information Sciences, Springer, Berlin, Germany, 2005.

[20] Z. Sun and S. S. Ge, Switched Linear Systems: Control and Design, Communications in Control Engineering, Springer, Berlin, Germany, 2005.

[21] D. Liberzon, Switching in Systems and Control, Systems \& Control: Foundations \& Applications, Birkhäuser, Boston, Mass, USA, 2003.

[22] M. de la Sen and A. Ibeas, "On the stability properties of linear dynamic time-varying unforced systems involving switches between parameterizations from topologic considerations via graph theory," Discrete Applied Mathematics, vol. 155, no. 1, pp. 7-25, 2007.

[23] J. D. Meiss, Differential Dynamical Systems, vol. 14 of Mathematical Modeling and Computation, SIAM, Philadelphia, Pa, USA, 2007.

[24] M. de la Sen and N. Luo, "A note on the stability of linear time-delay systems with impulsive inputs," IEEE Transactions on Circuits and Systems I, vol. 50, no. 1, pp. 149-152, 2003.

[25] K. Liu and L. Chen, "On a periodic time-dependent model of population dynamics with stage stucture and impulsive effects," Discrete Dynamics in Nature and Society, vol. 2008, Article ID 389727, 15 pages, 2008.

[26] Z. Zhao and X. Song, "On the study of chemostat model with pulsed input in a polluted environment," Discrete Dynamics in Nature and Society, vol. 2007, Article ID 90158, 12 pages, 2007.

[27] R. Shi and L. Chen, "Stage-structured impulsive SI model for pest management," Discrete Dynamics in Nature and Society, vol. 2007, Article ID 97608, 11 pages, 2007.

[28] H. Liu, H. Xu, J. Yu, and G. Zhu, "Stability results on age-structured SIS epidemic model with coupling impulsive effect," Discrete Dynamics in Nature and Society, vol. 2006, Article ID 83489, 11 pages, 2006.

[29] M. de la Sen and S. Alonso-Quesada, "A control theory point of view on Beverton-Holt equation in population dynamics and some of its generalizations," Applied Mathematics and Computation, vol. 199, no. 2 , pp. 464-481, 2008.

[30] M. de la Sen, "The generalized Beverton-Holt equation and the control of populations," Applied Mathematical Modelling, vol. 32, no. 11, pp. 2312-2328, 2008.

[31] M. de la Sen and S. Alonso-Quesada, "Model-matching-based control of the Beverton-Holt equation in ecology," Discrete Dynamics in Nature and Society, vol. 2008, Article ID 793512, 21 pages, 2008.

[32] M. de la Sen, "About the properties of a modified generalized Beverton-Holt equation in ecology models," Discrete Dynamics in Nature and Society, vol. 2008, Article ID 592950, 23 pages, 2008.

[33] X. Li and W. Yang, "Permanence of a discrete predator-prey systems with Beddington-DeAngelis functional response and feedback controls," Discrete Dynamics in Nature and Society, vol. 2008, Article ID 149267, 8 pages, 2008.

[34] Y.-H. Fan and L.-L. Wang, "Permanence for a discrete model with feedback control and delay," Discrete Dynamics in Nature and Society, vol. 2008, Article ID 945109, 8 pages, 2008.

[35] M. de la Sen, "The environment carrying capacity is not independent of the intrinsic growth rate for subcritical spawning stock biomass in the Beverton-Holt equation," Ecological Modelling, vol. 204, no. 1-2, pp. 271-273, 2007. 


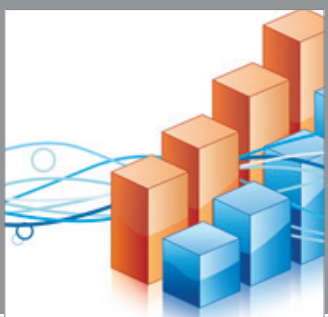

Advances in

Operations Research

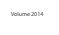

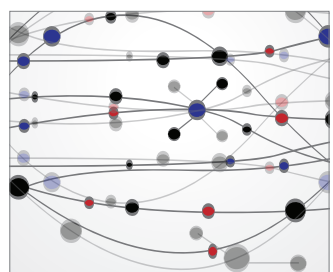

\section{The Scientific} World Journal
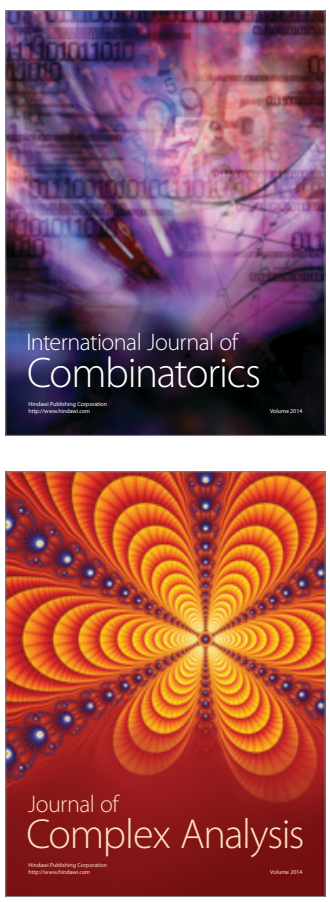

International Journal of

Mathematics and

Mathematical

Sciences
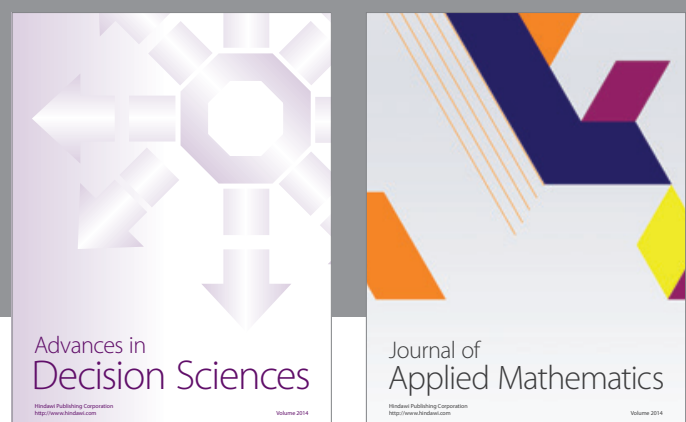

Journal of

Applied Mathematics
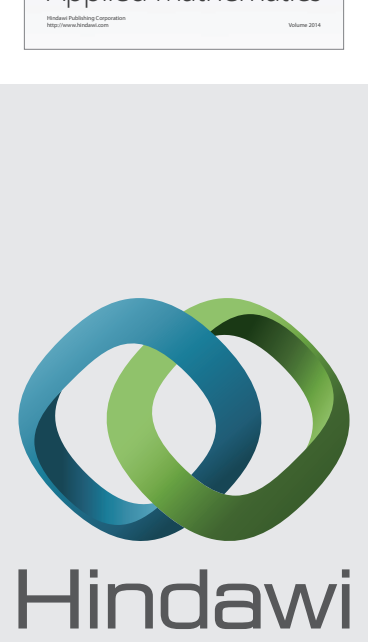

Submit your manuscripts at http://www.hindawi.com
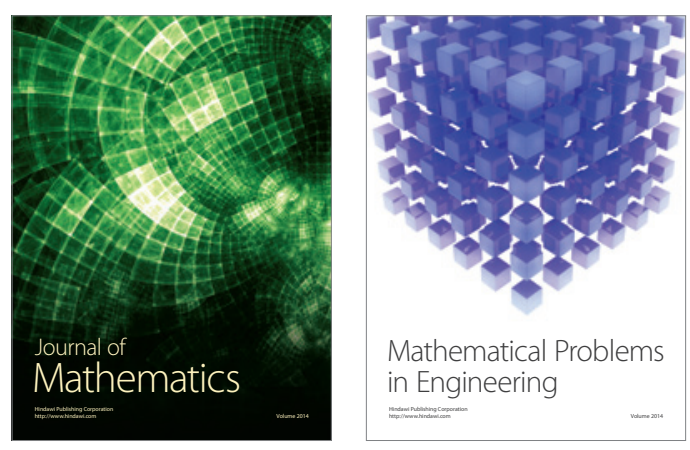

Mathematical Problems in Engineering
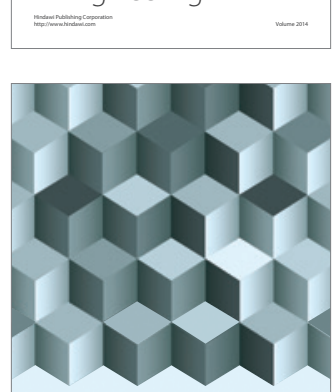

Journal of

Function Spaces
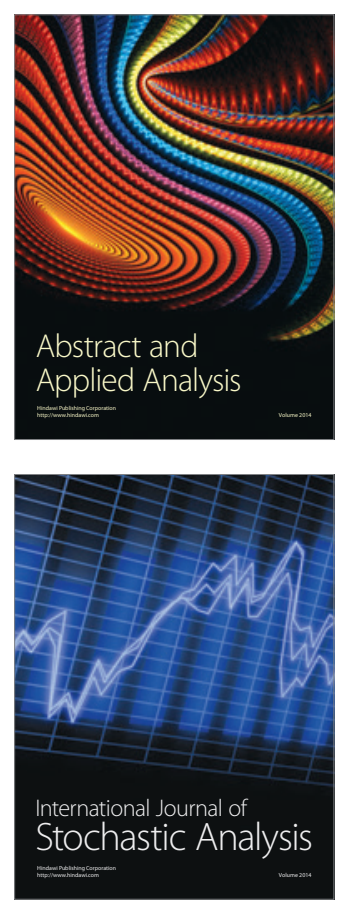

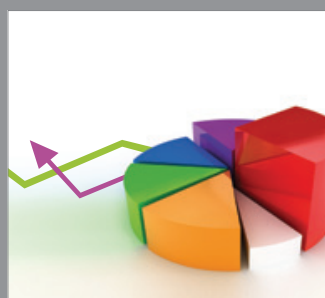

ournal of

Probability and Statistics

Promensencen
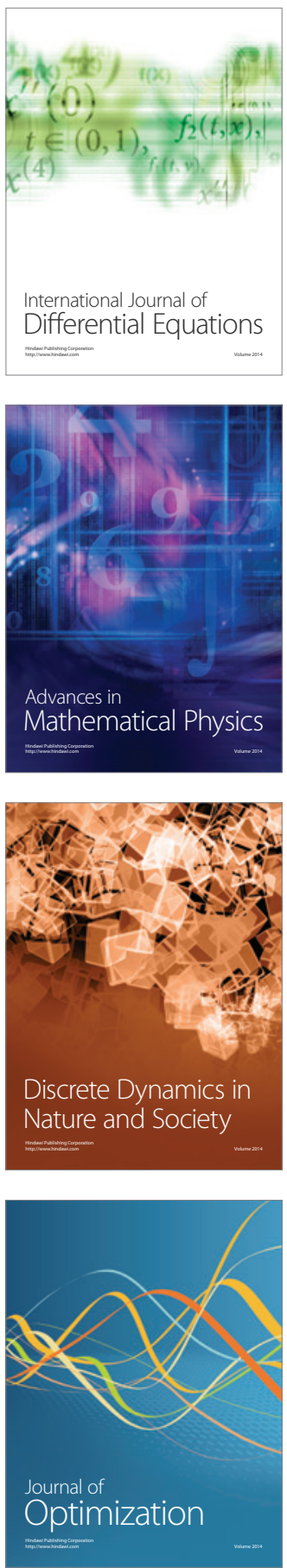